\title{
REVIEW ARTICLE OPEN Toward targeting inflammasomes: insights into their regulation and activation
}

\author{
Shelbi Christgen ${ }^{1}$, David E. Place ${ }^{1}$ and Thirumala-Devi Kanneganti iD $^{1}$
}

\begin{abstract}
Inflammasomes are multi-component signaling complexes critical to the initiation of pyroptotic cell death in response to invading pathogens and cellular damage. A number of innate immune receptors have been reported to serve as inflammasome sensors. Activation of these sensors leads to the proteolytic activation of caspase-1, a proinflammatory caspase responsible for the cleavage of proinflammatory cytokines interleukin-1 $\beta$ and interleukin-18 and the effector of pyroptotic cell death, gasdermin D. Though crucial to the innate immune response to infection, dysregulation of inflammasome activation can lead to the development of inflammatory diseases, neurodegeneration, and cancer. Therefore, clinical interest in the modulation of inflammasome activation is swiftly growing. As such, it is imperative to develop a mechanistic understanding of the regulation of these complexes. In this review, we divide the regulation of inflammasome activation into three parts. We discuss the transcriptional regulation of inflammasome components and related proteins, the post-translational mechanisms of inflammasome activation, and advances in the understanding of the structural basis of inflammasome activation.
\end{abstract}

Cell Research (2020) 30:315-327; https://doi.org/10.1038/s41422-020-0295-8

\section{INTRODUCTION}

Inflammasomes are signaling platforms crucial to the innate immune response to infectious diseases. These large, multimeric complexes form in response to molecular patterns unique to pathogens and cellular damage, triggering a cascade of downstream responses, including the induction of pyroptotic cell death and release of proinflammatory cytokines. ${ }^{1}$ Some inflammasomes directly recognize these patterns, while others indirectly sense these patterns through changes in the homeostatic environment of the cell. At the most simplistic level, an inflammasome is comprised of a sensor, an adapter, and an effector. Typically, inflammasome complexes are named after their sensor. The most well-established inflammasomes include the nucleotide-binding oligomerization domain-like receptor (NLR)-family, pyrin domain (PYD)-containing 1 (NLRP1); NLR-family, PYD-containing 3 (NLRP3); NLR-family apoptosis inhibitory protein (NAIP); NLR-family, caspase activation and recruitment domain (CARD)-containing 4 (NLRC4); absent in melanoma 2 (AIM2); and Pyrin inflammasomes. In addition, a number of other NLR-family proteins, including NLRP2, NLRP6, NLRP7, NLRP9, and NLRP12, among others, have been proposed to serve as sensors in inflammasome complexes. ${ }^{2-7}$ Several of these sensors use the apoptosis-associated speck-like protein containing a CARD (ASC) as an adapter molecule. The ASC adapter serves as a bridge, connecting the sensor to the downstream effector, caspase-1 (CASP1). After inflammasome assembly, CASP1 becomes activated through proximity-induced autoproteolysis. Active CASP1 is then free to process the immature forms of proinflammatory cytokines interleukin- $1 \beta$ $(\mathrm{IL}-1 \beta)$ and IL-18 into their mature signaling forms. ${ }^{8,9}$ Additionally, CASP1 cleaves the protein gasdermin D (GSDMD). ${ }^{10}$ After cleavage, the $\mathrm{N}$-terminal fragment of GSDMD (N-term GSDMD) oligomerizes to form pores in the cell membrane, allowing IL-1 $\beta$ and IL-18 to leave the cell and effectively executing pyroptotic, or inflammatory, cell death. ${ }^{10-13}$

Though these complexes serve vital functions in host defense, aberrant activation of inflammasomes has been linked with multiple diseases. Mutations within NLRP3 and Pyrin lead to the development of autoinflammatory diseases, including cryopyrinassociated periodic syndromes (CAPS) and familial Mediterranean fever (FMF). ${ }^{14-18}$ Additionally, chronic inflammasome activation has been tied to the development of metabolic syndromes, neurodegenerative diseases, and cancer progression. ${ }^{19-21}$ It is therefore unsurprising that inflammasome activation is a tightly regulated process. A number of transcription factors have been found to regulate the expression of inflammasome components, downstream effector molecules, and the upstream regulatory molecules required for successful activation. Furthermore, activation of each inflammasome is regulated by mechanisms unique to the sensing molecule. Broadly, these activation mechanisms can be divided into those reliant on direct ligand-binding and indirect mechanisms of activation. Recent structural studies have provided mechanistic details into the activation of different inflammasomes. Here, we break down the regulation of well-established inflammasomes into three separate areas to be discussed: the transcriptional regulation of inflammasome components, upstream regulators, and downstream effectors; the post-translational mechanisms of inflammasome activation; and the structural basis of inflammasome activation.

\section{TRANSCRIPTIONAL REGULATION OF INFLAMMASOMES}

Numerous proteins have been reported to be involved in the regulation of the inflammasome response to damage and infection.

${ }^{1}$ Department of Immunology, St. Jude Children's Research Hospital, Memphis, TN 38105, USA

Correspondence: Thirumala-Devi Kanneganti (thirumala-devi.kanneganti@stjude.org)

Received: 17 September 2019 Accepted: 20 February 2020

Published online: 9 March 2020 
Upstream Regulators: ZBP1, GBPs, IRGB10
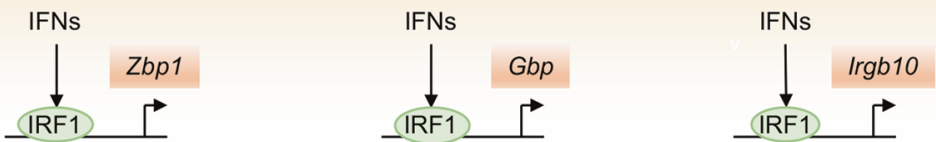

Sensors: AIM2, NLRC4, NAIPs, Pyrin, NLRP1, NLRP3
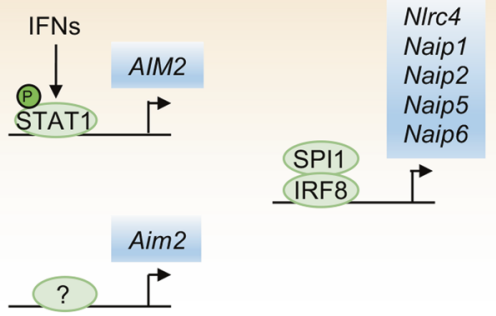

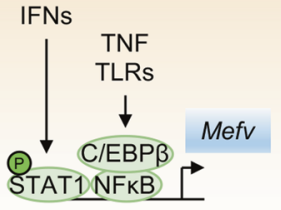

PERK PKA

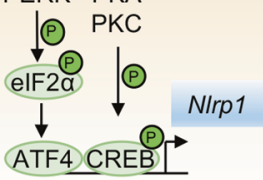

ATF4 CREB

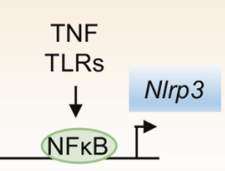

Adaptor: ASC

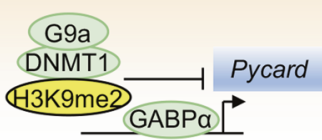

Caspases: CASP1, CASP4, CASP5, CASP11

IFN $\gamma$ IRF8) RRF1

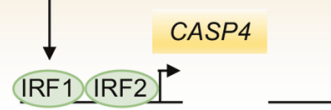

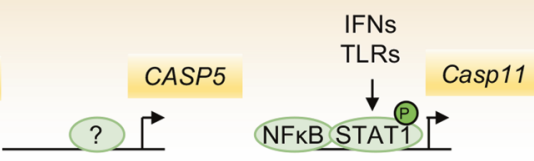

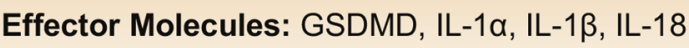
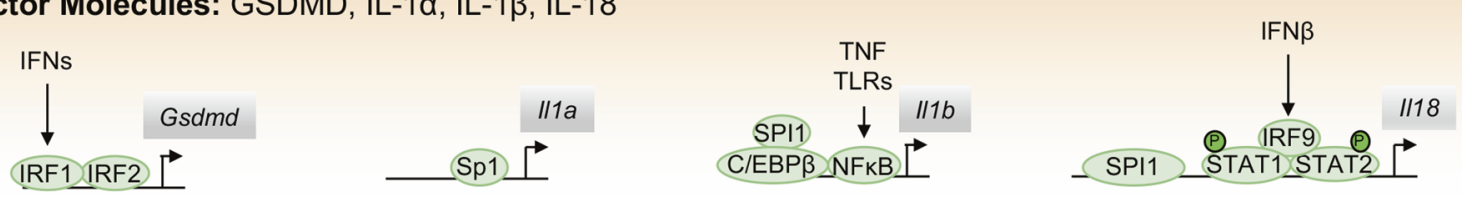

Fig. 1 Proposed transcriptional regulators of inflammasome components. Inflammasome activity is tightly regulated at the transcriptional level, and a number of factors have been proposed to regulate the expression of inflammasome components, molecules required upstream of activation, and downstream effector molecules.

Here, we discuss the transcriptional regulation of several proteins that are required upstream of inflammasome activation, directly participate in the inflammasome complex, or are crucial downstream effector molecules (Fig. 1).

Upstream regulatory molecules

Interferon regulatory factor 1 (IRF1) has emerged as a key factor regulating the transcription of a number of molecules upstream of the activation of several inflammasomes. One such molecule is the interferon (IFN)-inducible Z-DNA-binding protein 1 (ZBP1), also referred to as DNA-dependent activator of IFN-regulatory factors or DAl, which senses intracellular viruses and assembles a unique complex that mediates parallel necroptosis, apoptosis, and NLRP3mediated pyroptosis pathways. ${ }^{22}$ During influenza infection, Zbp1 is primarily upregulated by IRF1 following IFN production. ${ }^{23}$ Partial expression of $Z b p 1$, even in the absence of IRF1, suggests other factors may also play a role downstream of IFN signaling. During intracellular bacterial infection, IRF1 is required to upregulate a set of IFN-stimulated genes, including Gbp2, Gbp5, and Irgb10. These guanylate-binding proteins (GBPs) and immunity-related GTPase family member b10 (IRGB10) must be expressed to lyse the bacterial membrane or increase the exposure of transfected cytosolic lipopolysaccharide (LPS) or bacterial outer membrane vesicles for sensing by caspase-11 (CASP11) and downstream activation of the NLRP3 inflammasome. ${ }^{24-28}$ Additionally, these proteins are required to lyse intracellular bacteria such as Francisella novicida to free the bacterial dsDNA substrate for sensing by the AIM2 inflammasome. ${ }^{25,26}$ Immune recognition of the fungal pathogen Aspergillus fumigatus via activation of C-type lectin receptors (CTRs), Syk, and the nuclear factor kappa B (NF-KB) pathway leads to increased expression of Irf1. Concomitant recognition via Toll-like receptors (TLRs) is required for the TLR adapter MyD88 to associate with IRF1, mediating its translocation to the nucleus and the subsequent expression of Irgb10. Without both the CTR and TLR activation, IRGB10 is poorly expressed, and NLRP3 inflammasome activation is reduced during $A$. fumigatus infection. ${ }^{29}$

Components of the inflammasome complex

AIM2. In mice, AIM2 is constitutively expressed in macrophages, while in humans, it is upregulated by IFNs and signal transducer and activator of transcription 1 (STAT1) binding to a unique endogenous retroviral insertion sequence $-220 \mathrm{bp}$ upstream of AIM2. ${ }^{30}$ This variance in regulation likely plays an important role in differences between humans and mice that requires further investigation. As mentioned previously, transcriptional control of additional key upstream regulators of the AIM2 inflammasome further governs activation. For activation of the AIM2 inflammasome to occur during infection with intracellular bacteria such as F. novicida, IFNs must also upregulate IRF1 to induce GBPs and IRGB10 to carry out their roles in liberating bacterial ligands to be sensed by the inflammasomes. ${ }^{24-26}$ Together, multiple layers of IFN-mediated signaling tightly regulate activation of the AIM2 inflammasome. 
NAIPs and NLRC4. Until recently, no transcription factors were known to regulate the expression of the NAIP or NLRC4 proteins necessary for NLRC4 inflammasome activation. The transcription factor IRF8 (IFN regulatory factor 8), essential for development of multiple myeloid lineage cell types, has been found to be required for the basal expression of several murine NAIP proteins and murine NLRC4. Naip2 and Naip5 contain IRF8 consensus sequences at -590 and -390 bp upstream of their start sites, respectively, while NIrc4 contains an IRF8-binding site within an intronic sequence. ${ }^{31}$ Data from an earlier chromatin immunoprecipitation sequencing (ChIP-seq) study also found an enrichment of IRF8binding sites in the promoter regions of Naip2, Naip5, and Naip6, further supporting these findings. ${ }^{32}$ In addition to the IRF8 sites, SPI-1-binding sites are enriched in Naip1, Naip2, Naip5, and Naip6, suggesting SPI-1 and IRF8 may cooperate to regulate expression of key components of the NLRC4 inflammasome. ${ }^{33}$

Pyrin. The gene encoding the protein Pyrin, Mefv, is upregulated after LPS, tumor necrosis factor (TNF), or IFN treatment. ${ }^{34,35}$ Following stimulation with IFN $\gamma$, STAT1 binds to the gammaactivated sequence (GAS) element in the Mefv promoter at $-731 \mathrm{bp}$ and rapidly upregulates Mefv. ${ }^{34}$ TNF signaling was shown to promote human MEFV expression in a CCAAT enhancer-binding protein $\beta(\mathrm{C} / \mathrm{EBP} \beta)$ and NF-KB p65-dependent manner through binding of C/EBP $\beta$ and NF-KB to conserved sites at -163 and $-55 \mathrm{bp}$. In a mouse model of FMF, TNF signaling through TNF receptor 1 (TNFR1) was critical for driving pathogenic overexpression of Mefv, supporting earlier studies. ${ }^{36,37}$ Other putative transcription factor-binding sites have been identified but are poorly studied, including those for SPI-1, AP-1 (c-Jun/c-Fos), and Runt family transcription factors. ${ }^{34}$ Patients with mutations associated with FMF display varying severity of disease, and mutations in the promoter region (c. $-614 \mathrm{C}>\mathrm{G}$ or $\mathrm{c} .-382 \mathrm{C}>\mathrm{T}$ ) are associated with decreased or increased MEFV expression, respectively, suggesting that differential gene expression may play a clinically important role in FMF. ${ }^{38}$

NLRP1. Humans possess a single NLRP1, whereas mice have three paralogues, NLRP1 $a, b$, and $c$, with NLRP1c being $a$ pseudogene. In human cells, endoplasmic reticulum (ER) stress activates inositol-requiring enzyme 1 a (IRE1a) and protein kinase R-like ER kinase (PERK), which upregulates activating transcription factor 4 (ATF4), allowing it to bind the NLRP1 promoter and increase NLRP1 expression. ${ }^{39}$ Another study showed that protein kinase $A(P K A) / P K C$ and CAMP response element-binding protein (CREB) also regulated NLRP1 expression in myeloid leukemia cells; however, in HeLa cells this CREB-dependent NLRP1 expression was not observed, suggesting there may be cell type-specific regulation. ${ }^{39,40}$ In neurons, NLRP1 was repressed by heme oxygenase-1 (HO-1)-dependent inhibition of ATF4 expression, and overexpression of $\mathrm{HO}-1$ limited neuronal death and damage in a spinal cord injury model, supporting the proposed role for ATF4 in directly regulating NLRP1 expression. ${ }^{41}$

NLRP3. Numerous inflammatory diseases are driven by activation of the NLRP3 inflammasome, and a large number of upstream triggers are implicated in the transcriptional priming of NLRP3. Early studies identified a critical role for NF-KB in the upregulation of Nlrp3 following inflammatory signaling through the TLRs and their adapter proteins (MyD88, Toll-interleukin receptor domaincontaining adapter protein inducing IFN $\beta$ (TRIF)) and downstream kinases (IRAK1/4). ${ }^{42,43}$ The NF-KB-binding sites $(-1330$ to $-1292 \mathrm{bp}$ and -1238 to $-1228 \mathrm{bp}$ ) in the NIrp3 promoter were experimentally determined by electrophoretic mobility shift assay (EMSA) and ChIP assays. ${ }^{44}$ Additionally, signaling downstream of the TNF receptor, Fas-associated protein with death domain (FADD), and caspase-8 (CASP8) can also positively regulate NF-kB-dependent Nlrp3 transcription. ${ }^{42,43,45,46}$ A further study found that genetic loss of TNF in mice led to a downregulation of NLRP3 mRNA and the mRNA of other inflammasome components. Additionally, loss of TNF helped to protect against disease induced by NLRP3 mutations. ${ }^{47}$ While NF-KB-dependent transcriptional regulation of Nlrp3 is the most well studied, other putative transcription factorbinding sites have been identified in the promoter region including Sp1, c-Myb, AP-1, and c-Ets, and disruption of the Sp1 site leads to a reduction in NLRP3 expression. ${ }^{48}$ An additional study in human endothelial cells also found that NLRP3 was regulated by the sterol regulatory element-binding transcription factor 2 (SREBP2) binding between -1379 and -1368 bp. $^{49}$ Negative regulation of N/rp3 has also been observed following interaction of growth factor independence 1 (GFI1) with the promoter Gli-response element 1 (GRE1), which repressed expression of Nlrp3 and transcriptional activity of NF-KB p65. ${ }^{50}$ Post-transcriptionally, the expression of NLRP3 has been shown to be regulated by microRNAs. ${ }^{51,52}$ Given the diversity of activating signals for NLRP3, tight transcriptional regulation is likely an important checkpoint to preserve cell viability.

ASC. Multiple inflammasome sensors containing a PYD require the adapter protein ASC (encoded by Pycard) to recruit and activate CASP1. ASC is constitutively expressed in many cells and does not appear to be inducible by inflammatory signaling. Earlier studies identified ASC both through its ability to form protein aggregates during HL60 cell death, leading to the name ASC, and as a gene that is highly methylated in human breast cancers, leading to the name target of methylation-induced silencing (TMS1). ${ }^{53-55}$ Methylation-induced silencing of ASC was found to be important in many other cancer cell lines, and a recent study found that a long noncoding antisense RNA called PYCARD-AS1 localized to the PYCARD promoter, facilitating DNA methylation and $\mathrm{H} 3 \mathrm{~K} 9 \mathrm{me} 2$ modification via DNA methyltransferase 1 (DNMT1) and G9a recruitment. ${ }^{56-59}$ The ASC promoter is found within a large $\mathrm{CpG}$ island that is regulated by histone modifications. Basal expression of ASC appears to be regulated by the Ets family member GA-binding protein a (GABPa), which is enriched at a DNase I hypersensitive site (HS2) intronic element in the ASC promoter. ${ }^{60}$ Given the important role that ASC plays in inflammasome activation and, in some cases, apoptotic cell death, a better understanding of its regulation may provide insight into how cancers and autoinflammatory diseases progress.

\section{Caspases}

CASP1. The transcriptional regulation of CASP1 is poorly understood. In murine macrophages, CASP1 is constitutively expressed, with some reports suggesting that CASP1 may be upregulated by NF-KB or IRF1. Indeed, the Casp1 promoter contains putative NF$\mathrm{KB}-$ and IRF1-binding sites, and CASP1 is upregulated in chronic inflammatory conditions. ${ }^{61}$ Another study showed that IRF8 binding to an IRF8 consensus binding site at -40 to $-31 \mathrm{bp}$ upstream of the CASP1 start site regulated promoter activity in $\mathrm{B}$ cells, and that IRF1 synergistically acts with IRF8 to regulate CASP1 expression. ${ }^{62}$ Less well studied factors that regulate CASP1 include Ets1, p63, and p73. ${ }^{63-65}$ Given the critical role CASP1 plays in pyroptosis, it is important to understand how it is regulated at the basal level and in inflammatory diseases.

CASP4/5/11. Intracellular sensing of LPS is mediated by either caspases-4/5 in humans (CASP4 and CASP5, respectively) or CASP11 in mice. The expression of Casp11 can be induced by TLR4/TRIF-mediated activation of NF-KB or by IFN-mediated activation of STAT1. These molecules bind to putative sites at $-119 \mathrm{bp}$ and -17 to $-59 \mathrm{bp}$ in Casp 11, respectively. ${ }^{66-70}$ The NFKB-mediated regulation of Casp 11 expression after LPS stimulation was inhibited when poly(ADP-ribose) polymerase-1 (PARP1) was knocked down, suggesting that it acts as a transcriptional cofactor for NF-KB. ${ }^{71}$ Another proposed transcriptional regulator of Casp11 
is the C/EBP homologous protein (CHOP), which is important for upregulation following LPS-induced lung injury in mice. ${ }^{72}$

Human CASP4 and CASP5 both mediate pyroptosis downstream of recognition of intracellular LPS, but the relative contribution of each is still poorly understood. CASP4 and CASP5 proteins are constitutively expressed in monocytes, unlike the low basal expression of CASP11 in murine macrophages. ${ }^{73}$ In human HT-29 colon carcinoma cells, IFNy upregulated mRNA levels of CASP1 and CASP5, but not CASP4; however, CASP5 protein levels were not increased. In human monocyte THP-1 cells, CASP5 mRNA and protein levels were increased by LPS stimulation. ${ }^{74}$ Another study found that CASP5 was expressed in psoriatic skin and was regulated by IFN and NF-KB. ${ }^{75}$ In primary human monocytes, the mRNA of CASP4 and CASP5 was transiently increased by LPS, but the protein levels were not changed, suggesting that there are important cell type-specific mechanisms that regulate these proteins. ${ }^{73}$ More recently, a critical regulator of CASP4, IRF2 (IFN regulatory factor 2), was identified by CRISPR/Cas9 screening of human U937 monocyte cells and iPSC-derived monocytes. ${ }^{76}$ The loss of expression of CASP4 in IRF2 knockout cells could be rescued by IRF1-mediated transcription following priming with IFN ${ }^{76}{ }^{7}$ In a similar screening study, IRF2 and IRF1 both appeared to be dispensable for CASP4 expression in a human endothelial cell line EA.hy926, further suggesting that there is cell typespecific regulation of these LPS-responsive caspases. ${ }^{77}$

Downstream effector molecules

GSDMD. The executioner molecule of pyroptosis, GSDMD, is constitutively expressed and autoinhibited in myeloid cells until activation by caspase cleavage. Recently, the transcription factor IRF2 was identified as a key regulator of basal Gsdmd expression in murine macrophages. Similar to the IRF2-dependent regulation of human CASP4, IFNy priming of IRF2-deficient cells could rescue Gsdmd expression via IRF1. ${ }^{76,77}$ Basal GSDMD expression in U937 cells did not appear to require IRF1 or IRF2, in contrast with what was observed in the human endothelial cell line EA.hy926, but deletion of both IRF1/2 in U937 cells rendered them insensitive to IFNY-mediated upregulation of GSDMD. ${ }^{76}$ It is still unclear whether variation in the regulation of IRF2 and GSDMD, which has been identified between individuals, corresponds to differences in immune responses to Gram-negative bacteria and septic shock. ${ }^{78}$ Together, these studies establish a critical role for IRF1 and IRF2 in regulation of human GSDMD.

IL-1a, IL-1 $\beta, I L-18$. Inflammasome activation ultimately leads to lytic cell death and, in most cases, cleavage-induced maturation of the cytokines IL-1 $\beta$ and IL-18. Unlike IL-1 $\beta$, IL-1 a does not need to be cleaved to functionally signal through the IL-1 receptor (IL-1R). The human ILIA promoter contains an Sp1-binding site at -52 to $-45 \mathrm{bp}$ and an AP-1 site at -13 to $-5 \mathrm{bp}$ as well as NF-KB-binding sites that mediate expression in many cell types. ${ }^{79-82}$ An antisense transcript of IL-1a, AS-IL-1a, also positively regulates IL-1a expression by facilitating recruitment of RNA polymerase $\|$ to the $\| 1 a$ promoter. ${ }^{83}$ Rapamycin has been shown to negatively regulate the expression of IL-1a, disrupting inflammation associated with senescence. ${ }^{84}$ Associations between IL-1a single nucleotide polymorphisms (SNPs) and disease have been documented and are likely to influence the basal expression of this important inflammatory cytokine.

In contrast to IL-1a, which is expressed in many different cell types, IL-1 $\beta$ is expressed in myeloid cells in a highly inducible manner downstream of TLRs. ${ }^{85}$ The promoter of ILIB contains a classical TATA box (-31 bp), 2 putative CAAT boxes $(-125$ and $-75 \mathrm{bp}$ ), and binding sites for Sp1, SPI-1, C/EBP 3, CREB, and NF$\mathrm{KB}^{86-91}$ Similar to IL-1a, SNPs associated with disease progression have been identified in regulatory regions of IL-1 $\beta$ (National Center for Biotechnology Information), requiring future study into their contributions to diseases.
In addition to IL-1 $\beta$ and GSDMD, the cytokine IL-18 requires cleavage by CASP1 to become functional. IL-18 is constitutively expressed in murine bone marrow-derived macrophages (BMDMs) and human peripheral blood mononuclear cells (PBMCs), but can also be upregulated following IFN priming through STAT1/2 and IRF9. ${ }^{92-94}$ SNPs in the IL18 promoter are associated with inflammatory diseases, suggesting they lead to changes in gene transcriptional regulation. ${ }^{95,96}$ The expression of $\| 18$ in mice requires SPI-1 binding at -31 to $-13 \mathrm{bp}$ and IRF8 binding at -39 to $-22 \mathrm{bp}$, and IRF1 associates with IRF8 following IFNy stimulation. ${ }^{97,98}$ An AP-1 site was also identified at -1120 to $-1083 \mathrm{bp}$ that promotes IFNYinduced and basal expression of IL-18. ${ }^{85}$ Another study found that human IL-18 could be regulated by STAT5 binding at multiple promoter locations and that a potential Bcl6 repressor-binding site may compete for binding by STAT5 ${ }^{99,100}$ Additionaly, human IL-18 does not possess an IRF8 consensus binding site, suggesting it may be regulated differently than murine IL-18. ${ }^{101}$

\section{POST-TRANSLATIONAL MECHANISMS OF ACTIVATION}

Each inflammasome recognizes and becomes activated in response to unique signals. This can occur through a direct interaction between a ligand and the sensor of an inflammasome or indirectly through sensing of the cellular environment by an inflammasome. In addition, a number of post-translational modifications regulate the activation of the different inflammasome sensors. These posttranslational modifications are summarized in Table 1.

Activation of AIM2 and NAIP-NLRC4 by ligand binding The NAIP-NLRC4 and AIM2 inflammasomes are activated directly in response to the binding of a pathogen-associated ligand. Activation of the AIM2 and NAIP-NLRC4 inflammasomes is summarized in Fig. 2.

AIM2. The AIM2 inflammasome initiates pyroptosis in response to double-stranded DNA (dsDNA). The hematopoietic expression, IFN inducibility, nuclear localization (HIN)-200 domain of the AIM2 protein directly binds dsDNA within the cytosol, leading to the association of AIM2 with ASC and subsequent activation of the AIM2 inflammasome. ${ }^{102}$ Activation of AIM2 in response to intracellular bacteria requires IFN-inducible GTPases, including GBPs and IRGB10. ${ }^{26}$ These proteins target intracellular bacteria, leading to bacteriolysis and the freeing of dsDNA into the cytosol for sensing by AIM2.

NAIP-NLRC4. NAIPs and NLRC4 work cooperatively to mediate inflammasome activation. ${ }^{103-107}$ NAIPs are responsible for directly sensing bacterial ligands. ${ }^{103-105}$ Humans have a single NAIP capable of sensing the needle protein of the bacterial type 3 secretion system (T3SS) and flagellin proteins, while mice have several NAIPs. Like human NAIP, murine NAIP1 recognizes the needle protein of the T3SS, while murine NAIP2 recognizes the T3SS rod protein. NAIP5 and NAIP6 from mice recognize and bind bacterial flagellin proteins. ${ }^{103,104,107}$ The ligands of the remaining murine NAIPs remain unknown. The ligand-bound NAIP complex acts as a nucleation point by recruiting and activating NLRC4, which in turn activates CASP1. The CARD of NLRC4 allows direct interaction between NLRC4 and CASP1. However, NLRC4 is still capable of interacting with ASC and has been shown to localize to the ASC speck. ${ }^{108}$ In addition, some reports have found that flagellin leads to the phosphorylation of NLRC4 at Ser533, which primes NLRC4 for activation. ${ }^{109,110}$ In contrast, a recent study suggests that phosphorylation of Ser533 plays no role in the activation of NLRC4. ${ }^{111}$

Indirect activation of NLRP1 and Pyrin

In contrast to the AIM2 and NAIP-NLRC4 inflammasomes, the conformational changes that induce NLRP1 and Pyrin activation do 


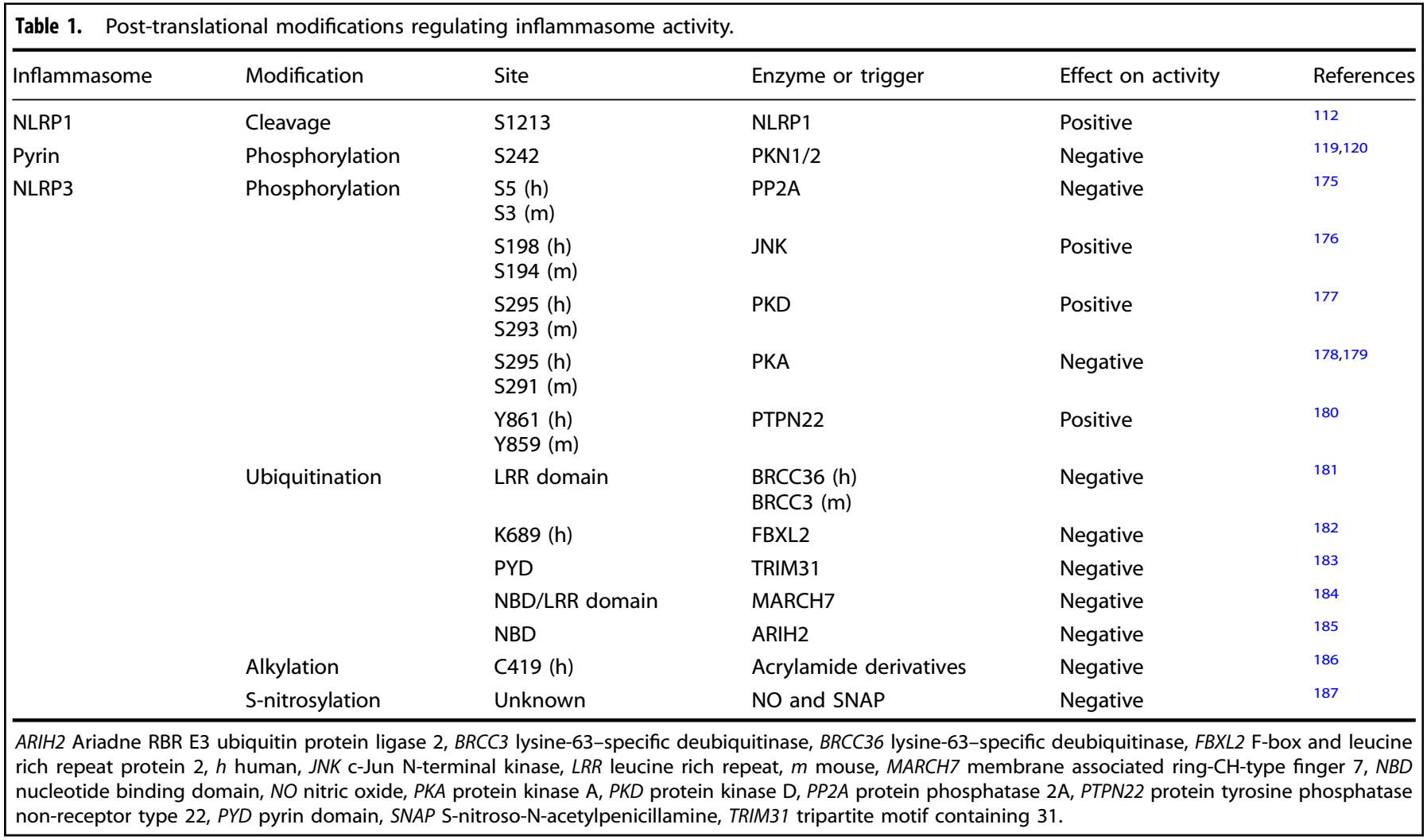

not occur as the result of direct ligand binding. The NLRP1 inflammasome is regulated through a unique process of functional degradation, while the Pyrin inflammasome senses the inactivation of other host proteins (Fig. 3).

NLRP1. NLRP1 was the first pattern recognition receptor that was found to be capable of forming an inflammasome complex. ${ }^{1}$ Encoded by a single NLRP1 gene in humans and three (NIrp1a-c) genes in mice, both human NLRP1 and the mouse paralogues possess a unique function to find (FIIND) domain. Self-cleavage at this FIIND domain in NLRP1 is required for later inflammasome function. ${ }^{112}$ Under normal cellular conditions, the two fragments of NLRP1 continue to interact, resulting in the sensor remaining autoinhibited. A subsequent cleavage event of the $\mathrm{N}$-terminus by a pathogenic trigger, such as cleavage of murine NLRP1b by lethal factor (LF) from Bacillus anthracis, results in ubiquitination and degradation of the now unstable fragment by the $\mathrm{E3}$ ubiquitin ligase UBR2. ${ }^{113-115}$ This functional degradation event relieves autoinhibition of the C-terminal fragment of the protein, allowing it to oligomerize and form an activated inflammasome complex. ${ }^{113-115}$ In addition, inhibition of host serine proteases DPP8/9 leads to a similar proteasomal-dependent activation of multiple variants of NLRP1, including those not sensitive to anthrax LF. ${ }^{16-118}$

Pyrin. Activation of the Pyrin inflammasome is also controlled without direct binding between Pyrin and a bacterial ligand. Under normal conditions, Pyrin is phosphorylated by protein kinase 1 (PKN1) and 2 (PKN2), and this phosphorylation event promotes the association of Pyrin with the 14-3-3 proteins, blocking Pyrin activation. ${ }^{119}$ Bacterial toxins, including the TcdB toxin from Clostridium difficile, inactivate host Rho-GTPase. ${ }^{120}$ Subsequently, PKN1 and PKN2 become inactivated, resulting in the loss of Pyrin interaction with $14-3-3$ proteins and inflammasome activation. ${ }^{119,120}$ Thus, the Pyrin inflammasome senses pathogens through the modifications of other host proteins.
Activation of NLRP3

Currently, there is no known unifying mechanism of activation for the NLRP3 inflammasome. It therefore remains unclear if NLRP3 is activated by a specific ligand or through indirect means. Numerous triggers have been reported to activate the NLRP3 inflammasome, including ATP, potassium efflux, lysosomal disruption by crystalline particulates, mitochondrial DNA, and the generation of reactive oxygen species..$^{121-125}$ Due to the sheer number of stimuli capable of activating NLRP3, and the differences in their structural and chemical properties, it is unlikely that a single ligand exists for this inflammasome. Instead, NLRP3 activation is likely regulated in an indirect manner reflecting the homeostatic nature of the cell. This notion is supported by a recent study establishing a novel regulator of NLRP3 activation, DDX3X, a protein normally involved in stress granule formation. ${ }^{126}$ By exploring the crosstalk between cellular stress and pyroptotic cell death, the authors found that DDX3X was required for NLRP3 activation and that sequestration of DDX3X by the formation of stress granules inhibited this inflammasome's activation. ${ }^{126}$ Thus, DDX3X represents a cellular life or death checkpoint, whereby the fate of the cell is decided by DDX3X's recruitment to either the stress granule or inflammasome complex. NLRP3's role as a homeostatic sensor is further supported by the apparent requirement for NEK7, a kinase normally involved in mitosis, in activation. ${ }^{127-129}$

Numerous models have been proposed to describe the common, upstream event that activates NLRP3 in response to such diverse stimuli. Potassium ion efflux has been shown to activate NLRP3 in response to an number of triggers and has been proposed to be the common mechanism of NLRP3 activation. ${ }^{130,131}$ Different activators, including the formation of membrane pores by toxins, facilitate potassium efflux capable of initiating NLRP3 activation. ${ }^{130,131}$ However, activation of NLRP3 through small molecules targeting mitochondria was found to be potassium efflux-independent. ${ }^{132}$ In addition, reactive oxygen species and oxidized mitochondrial 


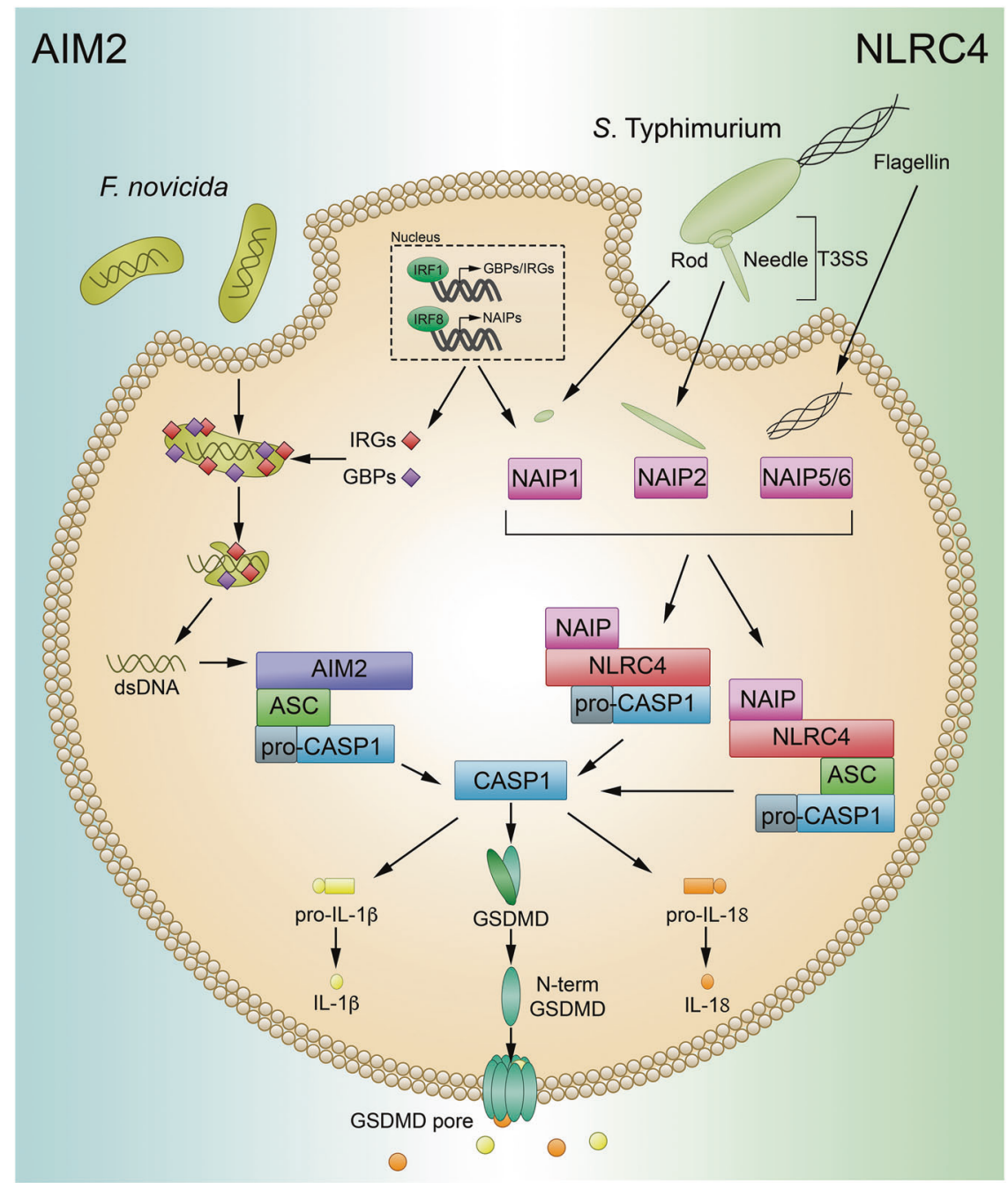

Fig. 2 Activation of AIM2 and NAIP-NLRC4 by direct ligand binding. AIM2: GBPs and IRGs mediate the bacteriolysis of intracellular bacteria, including F. novicida, leading to the exposure of dsDNA. Binding of dsDNA to AIM2 leads to AIM2 inflammasome activation. Active AIM2 recruits and activates CASP1 in an ASC-dependent manner to initiate pyroptosis through GSDMD and proinflammatory cytokine cleavage. NAIP-NLRC4: T3SS and flagellin proteins activate different murine NAIPs through direct binding interactions. NAIPs then activate NLRC4, which directly interacts with CASP1, leading to its autoactivation and the induction of pyroptosis.

DNA have been shown to activate the NLRP3 inflammasome, highlighting the importance of the mitochondria in NLRP3 activation. $^{123-125,133,134}$ Recently, disruption of the trans-Golgi network (TGN) has been described as a cellular event capable of activating NLRP3 in response to potassium-dependent and potassium-independent NLRP3 activators. ${ }^{135}$ The authors found that a polybasic stretch of four lysine residues on NLRP3 mediated charge-based interactions with phosphatidylinositol-4-phosphate, a component of the TGN. Interactions between these molecules led to the clustering of NLRP3 and subsequent assembly of ASC filaments at the disrupted TGN. ${ }^{135}$ These findings provide new avenues of exploration for the field as the mechanisms of the common upstream event responsible for NLRP3 activation continue to be sought.

In many circumstances, normal, basal levels of the NLRP3 protein within the cell are insufficient to trigger strong inflammasome activation. Thus, the current model of canonical NLRP3 activation requires a two-step process. Signal 1, known as the priming signal, leads to the transcriptional upregulation of NLRP3 inflammasome components and related molecules described earlier in this review, as well as post-translational modifications of NLRP3 that regulate activation (Table 1). After priming, a subsequent activating signal is required. At this point, NLRP3 oligomerizes and binds ASC, and inflammasome activation proceeds. FADD and CASP8 have been shown to play crucial, multifaceted roles in this two-step model. ${ }^{45}$

In addition to the large number of reported NLRP3 stimuli, there is an expansive cohort of live pathogens that activate the NLRP3 inflammasome through a number of distinct upstream pathways (Fig. 4). ${ }^{22,136-140}$ In the classical, or canonical pathway, activation of NLRP3 proceeds in the two-step manner. A. fumigatus appears to activate NLRP3 in murine macrophages through this canonical mechanism by initially engaging innate immune receptors to prime the cell, then activating the inflammasome after IRGB10depedent ligand release. ${ }^{29}$

Activation of NLRP3 by Gram-negative bacteria or cytosolic LPS requires a series of cellular events that are unique from canonical activation. Referred to as non-canonical, this mechanism of NLRP3 inflammasome activation is dependent on CASP11 in mice and CASP4 and CASP5 in humans. ${ }^{73,136}$ After activation by LPS, CASP4/ $5 / 11$ proceed to cleave GSDMD, triggering pyroptosis. ${ }^{73,141,142}$ The pores formed by CASP11-activated GSDMD lead to ionic flux in the cell, including potassium efflux, which induces NLRP3 activation, propagating the inflammatory signal. ${ }^{143}$ Similar to their role in liberating dsDNA for sensing by AIM2, IFN-regulated GBPs and IRGB10 are required in mice to effectively free LPS from 


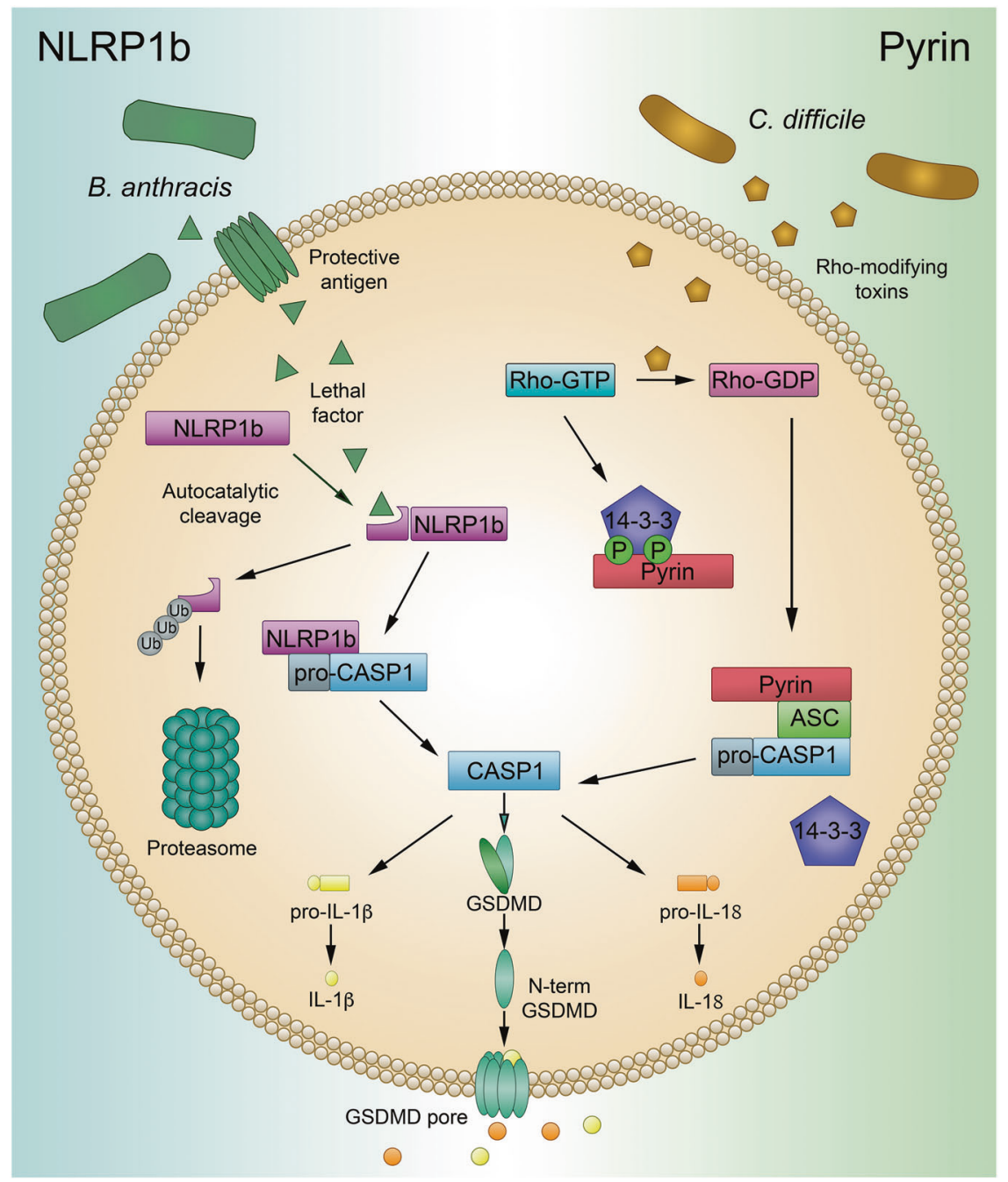

Fig. 3 Activation of NLRP1b and Pyrin through indirect mechanisms. NLRP1b: NLRP1b undergoes auto-processing at the FIIND domain to form two peptide fragments that continue to interact. NLRP1b-activating stimuli, such as LF from B. anthracis, cleave the N-terminal fragment of NLRP1b, targeting this fragment for ubiquitination and subsequent degradation. Degradation of the N-terminal fragment of NLRP1b relieves the C-terminal fragment from autoinhibition and allows the NLRP1b inflammasome to activate. Pyrin: Under homeostatic conditions, Pyrin is kept inactivated through phosphorylation-mediated interactions with 14-3-3. Rho-modifying toxins, such as TcdB from C. difficile, lead to the inactivation of Rho-GTPase. Loss of Rho-GTPase activity leads to unphosphorylated Pyrin. This allows Pyrin to disassociate from 14-3-3 and become active.

vacuole-resident or cytosolic intracellular Gram-negative bacteria for recognition by CASP $11 .{ }^{26}$

In the context of influenza infection, the innate immune sensor ZBP1 is emerging as a key regulator of the concurrent induction of NLRP3-dependent pyroptosis, mixed-lineage kinase domain-like pseudokinase (MLKL)-dependent necroptosis, and CASP8dependent apoptosis. Through RIP homotypic interaction motif (RHIM) domains, ZBP1 interacts with RIPK3 (receptor-interacting serine/threonine-protein kinase 3 ) and forms a multi-component complex that regulates the induction of these parallel cell death pathways. ${ }^{144}$ Though the specific ZBP1-activating ligands are currently unknown, recent studies have demonstrated that IFN-induced ZBP1 is required for influenza-induced NLRP3 activation. $22,145,146$

Transforming growth factor $\beta$-activated kinase 1 (TAK1) has been shown to control NLRP3 activation that appears to be independent of the canonical two-step activation, non-canonical CASP11-mediated activation, and IAV-induced ZBP1-mediated activation. Loss of TAK1 leads to the spontaneous activation of NLRP3 in a RIPK1-dependent manner, leading to the subsequent induction of cell death. ${ }^{147}$ Further studies have found that TAK1 inhibition after Yersinia infection leads to the CASP8-dependent cleavage of GSDMD and GSDME in a RIPK1-dependent manner. ${ }^{148-150}$ While there are still many unanswered questions regarding TAK1-mediated regulation of NLRP3 and cell death, these and other studies help to highlight the complexity of communication between cell death pathways. The unveiling of ZBP1 and TAK1 as master regulators of pyroptosis, apoptosis, and necroptosis, along with the rapidly expanding evidence of communication between these pathways, has led to the concept of PANoptosis, in which a putative PANoptosome acts as a central cell death complex that can initiate all three of these pathways. ${ }^{151,152}$ The convergence of these three programmed cell death pathways and the identification of master regulators controlling all three is intriguing and could be powerful therapeutically. Future research will be needed to determine the therapeutic potential of these master regulators and to unravel the complexities of this central PANoptotic process, including detailed exploration of the mechanisms of this multifaceted cell death. 


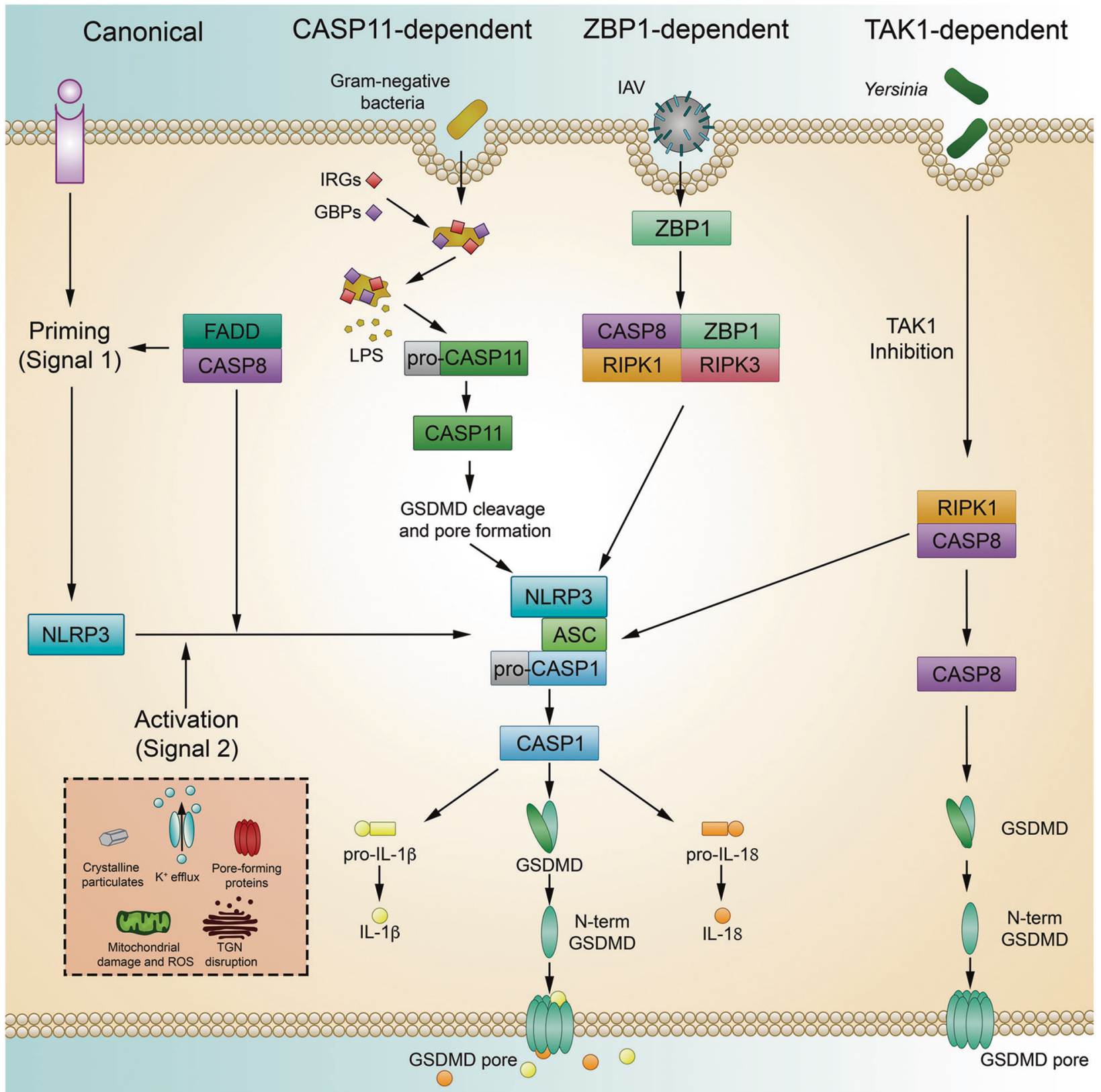

Fig. 4 Activation of the NLRP3 inflammasome. In response to a wide range of stimuli and live pathogens, NLRP3 becomes activated through canonical, CASP11-dependent, ZBP1-dependent, and TAK1-dependent mechanisms.

\section{STRUCTURAL BASIS OF INFLAMMASOME ACTIVITY}

At the molecular level, assembly of inflammasome signaling complexes is mediated through interactions between homotypic protein domains (Fig. 5). Several inflammasomes contain a PYD, responsible for the recruitment of the adapter protein ASC. After activation, inflammasomes utilizing this adapter will interact with ASC through PYD-PYD interactions, forming large, filamentous oligomers. ${ }^{153-155}$ ASC, comprised of an N-terminal CARD and Cterminal PYD, will then recruit CASP1 through CARD-CARD interactions. NLRP1 and NLRC4 are thought to directly interact with CASP1 through CARD-CARD interactions, though these NLRs could also interact with ASC through CARD-CARD interactions. Both PYD and CARD belong to the Death Domain (DD) superfamily and exhibit structural characteristics common to members of this family. ${ }^{156-158}$ The helical nature of members of the DD family leads to the formation of filamentous, higher-order complexes that allow for signal amplification and allosteric activation of executioner molecules, such as CASP1. In innate immunity, the propagation of signals is often mediated through the formation of these higher-order complexes, known as supramolecular organizational complexes (SMOCs). ${ }^{159,160}$ SMOCs utilize organellar membranes as scaffolds for formation and function by increasing the local concentration of signaling molecules to surpass a response threshold. ${ }^{159,160}$

Structural studies of inflammasome components by X-ray crystallography and nuclear magnetic resonance (NMR) have yielded a number of valuable insights. However, the heterogeneous, filamentous nature and size of the inflammasome complexes make analysis by these methods difficult. In recent years, advances in cryo-electron microscopy (cryo-EM) have led to a number of novel findings in the field of inflammasome activation. Such studies have revealed filamentous organization of PYDs and CARDs, ${ }^{154,161}$ plasticity within PYDs, ${ }^{162}$ and unique details of individual inflammasome activation. ${ }^{129,163,164}$ In addition, structural 


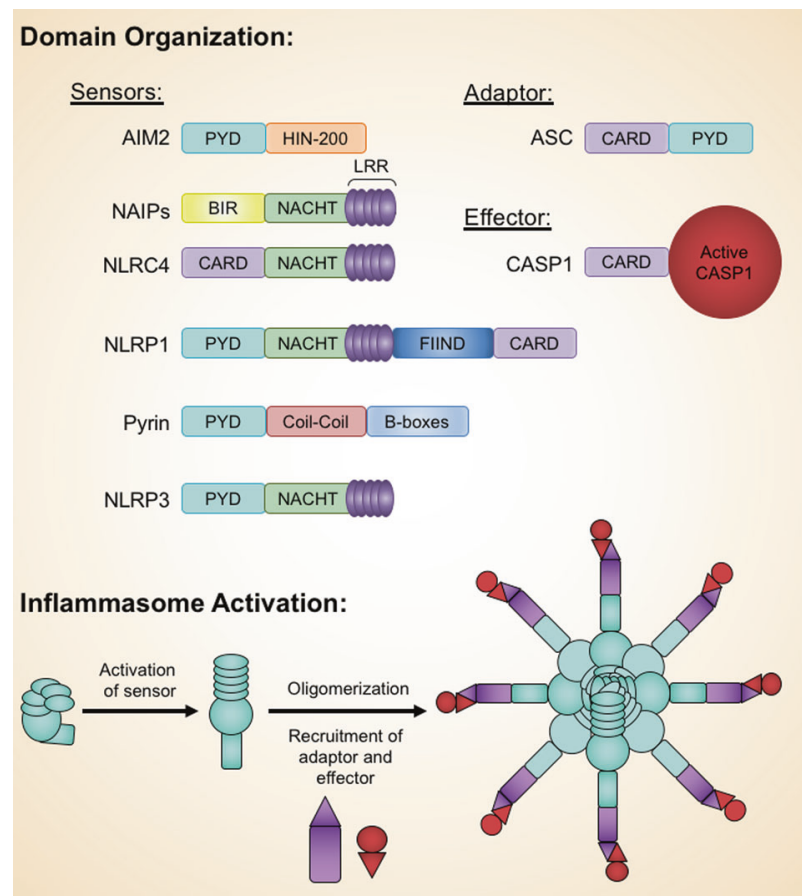

Fig. 5 Domain organization and basic inflammasome assembly. The domains of AIM2, NAIPs, NLRC4, NLRP1, Pyrin, NLRP3, ASC, and CASP1 are shown. The archetype inflammasome structure is comprised of a sensor, adapter, and effector protein. After activation, the sensor oligomerizes and recruits the adapter and effector proteins to the inflammasome complex. LRR, leucine rich repeat; BIR, baculoviral inhibitor of apoptosis protein repeat.

and biochemical studies have yielded mechanistic insight into the execution of pyroptotic cell death by gasdermin proteins.

Due to their more straightforward nature of activation, inflammasomes activated through direct ligand-binding mechanisms have presented attractive targets for structural researchers. As such, structural studies of the HIN-200 domain of AIM2 and the NAIP-NLRC4 inflammasome have yielded insights into the mechanistic details of activation.

\section{AIM2}

Activation of AIM2 relies on direct binding to dsDNA mediated through interactions between the DNA and the HIN domain of AIM2. Jin et al. reported these interactions to be mediated by electrostatic interactions between the positively charged HIN domain and the negatively charged phosphate backbone of the dsDNA, based on X-ray crystallography of the HIN domain complexed with DNA. ${ }^{165}$ The authors also found that activation of the AIM2 inflammasome, as measured by IL-1 $\beta$ secretion, was dependent on the length of the dsDNA ligand used in cellular experiments, and that DNA of around 80 base pairs was required for optimal IL- $1 \beta$ secretion. ${ }^{165}$ This evidence suggests that the DNA ligand, rather than the oligomerized sensor, serves as the structural scaffold for AIM2 inflammasome activation. A further structural study of a related murine Pyrin and HIN-containing (PYHIN) family member, p202, supports this hypothesis. ${ }^{166}$ p202 is comprised of a HIN1 and HIN2 domain and is known to inhibit AIM2 inflammasome activation. ${ }^{102}$ Yin et al. found that the HIN1 domain of p202 interacts with DNA, while the HIN2 domain mediates homotetramerization. Additionally, the authors found that the HIN2 domain is capable of interacting with the HIN domain of AIM2. The authors suggest that this interaction occludes clustering of AIM2 on the DNA, thereby preventing recruitment of the ASC adapter protein to the AIM2 inflammasome by spatially separating the PYDs of individual AIM2 molecules. ${ }^{166}$
NAIP-NLRC4

Cryo-EM studies have revealed that binding of NAIPs to their respective ligands triggers a conformational change that facilitates inflammasome activation. ${ }^{163,164,167,168}$ The observed rotation, occurring between helix domains within the NACHT domain, relieves autoinhibition by freeing a previously obscured NLRC4binding region. ${ }^{163,164,167,168}$ Binding of an NLRC4 molecule to this newly exposed, highly charged region on the activated NAIP triggers a similar rotation in the NLRC4 NACHT domain. As with the conformational change observed in NAIPs, this rotation exposes a charged NLRC4-binding site, thus propagating selfoligomerization. Therefore, the ligand-bound NAIP serves as a nucleation point for the formation of the activated wheel-shaped NAIP-NLRC4 inflammasome, where only a single activated NAIP subunit is complexed with multiple NLRC4 subunits through NACHT interactions.

NLRP3

The NLRP3 inflammasome has also been of high interest in the field of structural inflammasome biology for a number of years. In a recent study, Sharif et al. report a $3.8 \AA$ cryo-EM structure of inactive NLRP3 in complex with the mitotic kinase NEK7. ${ }^{129}$ Here, the authors found that electrostatic interactions between a predominantly negative NLRP3 interface and a positive NEK7 interface mediate NLRP3-NEK7 interactions, and they hypothesize that NEK7 plays a role in bridging NLRP3 protomers. Biochemical analysis of site-directed mutants supports these cryo-EM findings. As the structure of NLRP3/NEK7 is in an inactivated state, the authors incorporated the cryo-EM structure of activated NLRC4 to model an activated NLRP3 complex. Utilizing this predicted model, the authors hypothesize that while NEK7 is insufficient to induce activation, it may facilitate NLRP3 oligomerization by bridging NLRP3 protomers. This first structural look at the NLRP3 inflammasome also provides valuable insight into the potential effects of CAPS-associated mutations. Future studies investigating activated NLRP3 and the effects of CAPS-related mutations on the structural assembly of the NLRP3 inflammasome will provide valuable therapeutic insight into CAPS pathogenesis.

\section{Gasdermin}

The X-ray crystal structure of full-length GSDMA3 shows that gasdermins are kept autoinhibited through interactions between $\mathrm{N}$ - and C-terminal domains. ${ }^{10}$ Cleavage relieves this autoinhibition, freeing the cytotoxic $\mathrm{N}$-terminal fragment to oligomerize and form pores of $\approx 18 \mathrm{~nm}$ in the cell membrane. Biochemical experiments and analysis of the cryo-EM structure of the activated GSDMA3 pore determined that interactions between GSDMA3 and acidic lipid head groups are necessary for pore formation. ${ }^{169}$ The cryoEM density of a non-membrane-inserted pore was observed in this study, prompting the authors to propose a model of activation whereby the $\mathrm{N}$-terminal fragments oligomerize before insertion into the membrane. ${ }^{169}$ It remains to be determined if this observed "soluble pore" is an in vitro artifact of purification or a physiologically relevant intermediate state of the gasdermin pore. In contrast, Mulvihill et al. found that insertion of the N-terminal fragment of human GSDMD into lipid membranes precedes oligomerization and pore formation. ${ }^{170}$ Further dissection of the mechanistic order of events in gasdermin membrane pore formation will profoundly impact our understanding of the functionality of gasdermin family proteins.

\section{CONCLUDING REMARKS}

The regulation of inflammasomes is a delicately complex process, mediated by a wide number of transcription factors, regulatory molecules, and cellular pathways. Activation of inflammasomes plays a critical role in the defense of a host against infection and sterile insults, but dysregulation of any of these regulatory 
processes can lead to the development and progression of disease. As such, the efficacy of therapies targeting the upstream activation of inflammasomes and the downstream consequences of inflammasome activation is being avidly explored in a number of diseases. A recent study in mice found that inducing the activation of NLRP3 in conjunction with immune checkpoint inhibitor therapy is beneficial against tumors. ${ }^{171}$ Conversely, a phase 3 clinical study of the drug canakinumab found that blocking IL-1 $\beta$ can reduce the incidences of cardiovascular disease and lung cancer. ${ }^{172,173}$ In addition to these studies, a number of other clinical trials exploring the role of $\mathrm{IL}-1$ signaling and inflammasome activation are currently in progress or recruiting. The different outcomes of these studies highlight the contextspecific roles of inflammasome activation and IL-1 signaling in different diseases, indicating that thorough future studies are required to unlock the therapeutic potential of inflammasome modulation. From a therapeutic standpoint, the unique characteristics of each inflammasome's mechanism of activation are promising. The ability to selectively target an aberrant inflammasome, for example the Pyrin inflammasome in patients with FMF or the NLRP3 inflammasome in patients with CAPS, while leaving the others free to respond to infections and other insults, presents an attractive alternative to a total blockade of IL-1 $\beta$ signaling or inhibiting a shared downstream effector molecule. As such, it is crucial to develop a thorough mechanistic understanding of each of the inflammasomes.

While our understanding of the mechanisms regulating inflammasome activation has expanded tremendously, many crucial questions remain unanswered. Though it is one of the most well-studied inflammasomes, there is not a universally agreed upon model of NLRP3 activation. In addition, it is unclear what innate immune sensors, in addition to the ones discussed in this review, form true inflammasome complexes. Several reports suggest that multiple sensors can be recruited to the same complex. ${ }^{108,174}$ While the functional significance of heterogeneous inflammasome complexes is unclear, the concept is intriguing and will likely produce interesting studies in the future.

Further structural studies of inflammasome biology will also provide valuable insight into the conserved and unique mechanisms of inflammasome activation. It is currently unclear how the oligomerized sensors and filamentous adapter and effector regions of inflammasomes spatially interact with one another, as current structures do not include both of these regions. Future studies will likely focus on resolving these interactions. Furthermore, currently available structures suggest that in addition to having unique mechanisms of activation, different inflammasomes also have unique mechanisms of assembly. Developing a mechanistic understanding of the activation and assembly of each inflammasome at the transcriptional, post-translational, and structural levels will help guide the development of therapeutics and future clinical trials.

\section{ACKNOWLEDGEMENTS}

The authors acknowledge many investigators in the field whose primary data could not be cited in this review because of space limitations. The authors would like to thank members of the Kanneganti lab for helpful feedback during the editing of this review. Work in the Kanneganti lab is supported by the National Institutes of Health grants CA163507, AR056296, Al124346, and Al101935 and by ALSAC to T.-D.K.

\section{AUTHOR CONTRIBUTIONS}

S.C., D.E.P., and T.-D.K. contributed to the gathering of information and writing and editing of this review.

\section{ADDITIONAL INFORMATION}

Competing interests: The authors declare no competing interests.

\section{REFERENCES}

1. Martinon, F., Burns, K. \& Tschopp, J. The inflammasome: a molecular platform triggering activation of inflammatory caspases and processing of prolL- $\beta$. Mol. Cell. 10, 417-426 (2002).

2. Janowski, A. M., Sutterwala, F. S. A typical inflammasomes. In: Di Virgilio, F., Pelegrín, P., eds. NLR Proteins: Methods and Protocols. Methods in Molecular Biology. New York, NY: Springer New York; 2016:45-62.

3. Minkiewicz, J., Vaccari, J. P., de, R \& Keane, R. W. Human astrocytes express a novel NLRP2 inflammasome. Glia 61, 1113-1121 (2013).

4. Levy, M. et al. Microbiota-modulated metabolites shape the intestinal microenvironment by regulating NLRP6 inflammasome signaling. Cell 163, 1428-1443 (2015).

5. Khare, S. et al. An NLRP7-containing inflammasome mediates recognition of microbial lipopeptides in human macrophages. Immunity 36, 464-476 (2012).

6. Zhu, S. et al. Nlrp9b inflammasome restricts rotavirus infection in intestinal epithelial cells. Nature 546, 667-670 (2017).

7. Vladimer, G. I. et al. The NLRP12 inflammasome recognizes Yersinia pestis. Immunity 37, 96-107 (2012).

8. Thornberry, N. A. et al. A novel heterodimeric cysteine protease is required for interleukin-1 beta processing in monocytes. Nature 356, 768-774 (1992).

9. Howard, A. D. et al. IL-1-converting enzyme requires aspartic acid residues for processing of the IL-1 beta precursor at two distinct sites and does not cleave 31-kDa IL-1 alpha. J. Immunol.147, 2964-2969 (1991).

10. Ding, J. et al. Pore-forming activity and structural autoinhibition of the gasdermin family. Nature 535, 111-116 (2016).

11. Liu X. et al. Inflammasome-activated gasdermin D causes pyroptosis by forming membrane pores. Nature 535, 153-158 (2016).

12. Aglietti, R. A. et al. GsdmD p30 elicited by caspase-11 during pyroptosis forms pores in membranes. Proc. Natl. Acad. Sci. USA 113, 7858-7863 (2016).

13. Sborgi, L. et al. GSDMD membrane pore formation constitutes the mechanism of pyroptotic cell death. EMBO J. 35, 1766-1778 (2016).

14. French FMF Consortium A candidate gene for familial Mediterranean fever. Nat. Genet. 17, 25-31 (1997).

15. The International FMF Consortium. Ancient missense mutations in a new member of the RoRet gene family are likely to cause familial Mediterranean fever. Cell 90, 797-807 (1997).

16. Hoffman, H. M., Mueller, J. L., Broide, D. H., Wanderer, A. A. \& Kolodner, R. D. Mutation of a new gene encoding a putative pyrin-like protein causes familial cold autoinflammatory syndrome and Muckle-Wells syndrome. Nat. Genet. 29, 301-305 (2001).

17. Neven, B. Molecular basis of the spectral expression of CIAS1 mutations associated with phagocytic cell-mediated autoinflammatory disorders CINCA/ NOMID, MWS, and FCU. Blood 103, 2809-2815 (2004).

18. Feldmann, J. et al. Chronic infantile neurological cutaneous and articular syndrome is caused by mutations in CIAS1, a gene highly expressed in polymorphonuclear cells and chondrocytes. Am. J. Hum. Genet. 71, 198-203 (2002).

19. Wen, H., Ting, J. P.-Y. \& O'Neill, L. A. J. A role for the NLRP3 inflammasome in metabolic diseases-did Warburg miss inflammation? Nat. Immunol. 13, 352-357 (2012).

20. Voet, S., Srinivasan, S., Lamkanfi, M. \& van Loo, G. Inflammasomes in neuroinflammatory and neurodegenerative diseases. EMBO Mol. Med. 11, e10248 (2019).

21. Karki, R. \& Kanneganti, T.-D. Diverging inflammasome signals in tumorigenesis and potential targeting. Nat. Rev. Cancer 19, 197-214 (2019).

22. Kuriakose, T. et al. ZBP1/DAI is an innate sensor of influenza virus triggering the NLRP3 inflammasome and programmed cell death pathways. Sci. Immunol. 1, aag2045 (2016).

23. Kuriakose, T., Zheng, M., Neale, G. \& Kanneganti, T.-D. IRF1 Is a transcriptional regulator of ZBP1 promoting NLRP3 inflammasome activation and cell death during influenza virus infection. J. Immunol. 200, 1489-1495 (2018).

24. Man, S. M. et al. The transcription factor IRF1 and guanylate-binding proteins target activation of the AIM2 inflammasome by Francisella infection. Nat. Immunol. 16, 467-475 (2015).

25. Meunier, E. et al. Guanylate-binding proteins promote activation of the AIM2 inflammasome during infection with Francisella novicida. Nat. Immunol. 16, 476-484 (2015).

26. Man, S. M. et al. IRGB10 liberates bacterial ligands for sensing by the AIM2 and caspase-11-NLRP3 inflammasomes. Cell 167, 382-396.e17 (2016).

27. Briken, V. et al. Interferon regulatory factor 1 is required for mouse Gbp gene activation by gamma interferon. Mol. Cell Biol. 15, 975-982 (1995).

28. Santos, J. C. et al. LPS targets host guanylate-binding proteins to the bacterial outer membrane for non-canonical inflammasome activation. EMBO J. 37, e98089 (2018). 
29. Briard, B. et al. Fungal ligands released by innate immune effectors promote inflammasome activation during Aspergillus fumigatus infection. Nat. Microbiol. 4, 316-327 (2019)

30. Chuong, E. B., Elde, N. C. \& Feschotte, C. Regulatory evolution of innate immunity through co-option of endogenous retroviruses. Science $\mathbf{3 5 1}$, 1083-1087 (2016).

31. Karki, R. et al. IRF8 regulates transcription of Naips for NLRC4 inflammasome activation. Cell 173, 920-933.e13 (2018).

32. Olsson, A. et al. Single-cell analysis of mixed-lineage states leading to a binary cell fate choice. Nature 537, 698-702 (2016).

33. Langlais, D., Barreiro, L. B. \& Gros, P. The macrophage IRF8/IRF1 regulome is required for protection against infections and is associated with chronic inflammation. J. Exp. Med. 213, 585-603 (2016).

34. Centola, M. et al. The gene for familial Mediterranean fever, MEFV, is expressed in early leukocyte development and is regulated in response to inflammatory mediators. Blood 95, 3223-3231 (2000).

35. Matzner, Y. et al. Expression of the familial Mediterranean fever gene and activity of the C5a inhibitor in human primary fibroblast cultures. Blood 96, 727-731 (2000)

36. Papin, S. et al. The tumor necrosis factor alpha-dependent activation of the human mediterranean fever (MEFV) promoter is mediated by a synergistic interaction between C/EBP beta and NF kappaB p65. J. Biol. Chem. 278, 48839-48847 (2003)

37. Sharma, D., Malik, A., Guy, C., Vogel, P. \& Kanneganti, T.-D. TNF/TNFR axis promotes pyrin inflammasome activation and distinctly modulates pyrin inflammasomopathy. J. Clin. Investig. 129, 150-162 (2019).

38. Notarnicola, C. et al. Characterization of new mutations in the $5^{\prime}$-flanking region of the familial Mediterranean fever gene. Genes Immun. 10, 273-279 (2009).

39. D'Osualdo, A. et al. Transcription factor ATF4 induces NLRP1 inflammasome expression during endoplasmic reticulum stress. PLoS One 10, e0130635 (2015).

40. Sanz, C. et al. NALP1 is a transcriptional target for CAMP-response-elementbinding protein (CREB) in myeloid leukaemia cells. Biochem. J. 384, 281-286 (2004).

41. Lin, W.-P. et al. Heme oxygenase-1 promotes neuron survival through downregulation of neuronal NLRP1 expression after spinal cord injury. J. Neuroinflammation 13, 52 (2016).

42. Bauernfeind, F. G. et al. Cutting edge: NF-kappaB activating pattern recognition and cytokine receptors license NLRP3 inflammasome activation by regulating NLRP3 expression. J. Immunol. 183, 787-791 (2009).

43. Fernandes-Alnemri, T., Yu, J.-W., Datta, P., Wu, J. \& Alnemri, E. S. AIM2 activates the inflammasome and cell death in response to cytoplasmic DNA. Nature 458 , 509-513 (2009).

44. Qiao, Y., Wang, P., Qi, J., Zhang, L. \& Gao, C. TLR-induced NF-KB activation regulates NLRP3 expression in murine macrophages. FEBS Lett. 586, 1022-1026 (2012).

45. Gurung, P. et al. FADD and Caspase- 8 Mediate Priming and Activation of the Canonical and Noncanonical Nlrp3 Inflammasomes. J. Immunol. 192, 1835-1846 (2014).

46. Franchi, L. et al. Cytosolic flagellin requires Ipaf for activation of caspase- 1 and interleukin $1 \beta$ in salmonella-infected macrophages. Nat. Immunol. 7, 576-582 (2006).

47. McGeough, M. D. et al. TNF regulates transcription of NLRP3 inflammasome components and inflammatory molecules in cryopyrinopathies. J. Clin. Investig. 127, 4488-4497 (2017).

48. Anderson, J. P. et al. Initial description of the human NLRP3 promoter. Genes Immun. 9, 721-726 (2008).

49. Xiao, $\mathrm{H}$. et al. Sterol regulatory element binding protein 2 activation of NLRP3 inflammasome in endothelium mediates hemodynamic-induced atherosclerosis susceptibility. Circulation 128, 632-642 (2013).

50. Zhu, L. et al. The transcription factor GFI1 negatively regulates NLRP3 inflammasome activation in macrophages. FEBS Lett. 588, 4513-4519 (2014).

51. Bauernfeind, F. et al. NLRP3 inflammasome activity is negatively controlled by miR-223. J. Immunol. 189, 4175-4181 (2012).

52. Haneklaus, M. et al. Cutting edge: miR-223 and EBV miR-BART15 regulate the NLRP3 inflammasome and IL-1 $\beta$ production. J. Immunol. 189, 3795-3799 (2012).

53. Stimson, K. M. \& Vertino, P. M. Methylation-mediated silencing of TMS1/ASC is accompanied by histone hypoacetylation and CpG island-localized changes in chromatin architecture. J. Biol. Chem. 277, 4951-4958 (2002).

54. Conway, K. E. et al. TMS1, a novel proapoptotic caspase recruitment domain protein, is a target of methylation-induced gene silencing in human breast cancers. Cancer Res. 60, 6236-6242 (2000).

55. Masumoto, J. et al. ASC, a novel 22-kDa protein, aggregates during apoptosis of human promyelocytic leukemia HL-60 cells. J. Biol. Chem. 274, 33835-33838 (1999).
56. Riojas, M. A. et al. Methylation-induced silencing of ASC/TMS1, a pro-apoptotic gene, is a late-stage event in colorectal cancer. Cancer Biol. Ther. 6, 1710-1716 (2007).

57. Miao, H. et al. A long noncoding RNA distributed in both nucleus and cytoplasm operates in the PYCARD-regulated apoptosis by coordinating the epigenetic and translational regulation. PLoS Genet. 15, e1008144 (2019).

58. Moriai, R. et al. A proapoptotic caspase recruitment domain protein gene, TMS1, is hypermethylated in human breast and gastric cancers. Anticancer Res. 22, 4163-4168 (2002).

59. Stone, A. R. et al. Aberrant methylation and down-regulation of TMS1/ASC in human glioblastoma. Am. J. Pathol. 165, 1151-1161 (2004).

60. Lucas, M. E., Crider, K. S., Powell, D. R., Kapoor-Vazirani, P. \& Vertino, P. M. Methylation-sensitive regulation of TMS1/ASC by the Ets factor, GA-binding protein-alpha. J. Biol. Chem. 284, 14698-14709 (2009).

61. Lee, D.-J. et al. Regulation and function of the caspase-1 in an inflammatory microenvironment. J. Investig. Dermatol. 135, 2012-2020 (2015).

62. Lv, D.-W., Zhang, K. \& Li, R. Interferon regulatory factor 8 regulates caspase-1 expression to facilitate Epstein-Barr virus reactivation in response to $B$ cell receptor stimulation and chemical induction. PLoS Pathog. 14, e1006868 (2018).

63. Jain, N., Sudhakar, C. \& Swarup, G. Tumor necrosis factor-alpha-induced caspase1 gene expression. Role of p73. FEBS J. 274, 4396-4407 (2007).

64. Celardo, I. et al. Caspase-1 is a novel target of p63 in tumor suppression. Cell Death Dis. 4, e645 (2013).

65. Pei, $\mathrm{H}$. et al. Caspase-1 is a direct target gene of ETS1 and plays a role in ETS1induced apoptosis. Cancer Res. 65, 7205-7213 (2005).

66. Meunier, E. \& Broz, P. Interferon-induced guanylate-binding proteins promote cytosolic lipopolysaccharide detection by caspase-11. DNA Cell Biol. 34, 1-5 (2015).

67. Schauvliege, R., Vanrobaeys, J., Schotte, P. \& Beyaert, R. Caspase-11 gene expression in response to lipopolysaccharide and interferon-gamma requires nuclear factor-kappa B and signal transducer and activator of transcription (STAT) 1. J. Biol. Chem. 277, 41624-41630 (2002).

68. Rathinam, V. A. K. et al. TRIF licenses caspase-11-dependent NLRP3 inflammasome activation by gram-negative bacteria. Cell 150, 606-619 (2012).

69. Yen, J.-H. \& Ganea, D. Interferon beta induces mature dendritic cell apoptosis through caspase-11/caspase-3 activation. Blood 114, 1344-1354 (2009).

70. Gurung, P. et al. Toll or interleukin-1 receptor (TIR) domain-containing adaptor inducing interferon- $\beta$ (TRIF)-mediated caspase-11 protease production integrates Toll-like receptor 4 (TLR4) protein- and NIrp3 inflammasome-mediated host defense against enteropathogens. J. Biol. Chem. 287, 34474-34483 (2012).

71. Yoo, L., Hong, S., Shin, K. S. \& Kang, S. J. PARP-1 regulates the expression of caspase-11. Biochem. Biophys. Res. Commun. 408, 489-493 (2011).

72. Endo, M., Mori, M., Akira, S. \& Gotoh, T. C/EBP homologous protein (CHOP) is crucial for the induction of caspase-11 and the pathogenesis of lipopolysaccharide-induced inflammation. J. Immunol. 176, 6245-6253 (2006).

73. Viganò, E. et al. Human caspase- 4 and caspase- 5 regulate the one-step noncanonical inflammasome activation in monocytes. Nat. Commun. 6, 8761 (2015).

74. Lin, X. Y., Choi, M. S. \& Porter, A. G. Expression analysis of the human caspase-1 subfamily reveals specific regulation of the CASP5 gene by lipopolysaccharide and interferon-gamma. J. Biol. Chem. 275, 39920-39926 (2000).

75. Salskov-Iversen, M. L., Johansen, C., Kragballe, K. \& Iversen, L. Caspase-5 expression is upregulated in lesional psoriatic skin. J. Investig. Dermatol. 131, 670-676 (2011).

76. Benaoudia, S. et al. A genome-wide screen identifies IRF2 as a key regulator of caspase-4 in human cells. EMBO Rep. 20, e48235 (2019).

77. Kayagaki, N. et al. IRF2 transcriptionally induces GSDMD expression for pyroptosis. Sci. Signal. 12, eaax4917 (2019).

78. Fairfax, B. P. et al. Innate immune activity conditions the effect of regulatory variants upon monocyte gene expression. Science 343, 1246949 (2014).

79. McDowell, T. L., Symons, J. A. \& Duff, G. W. Human interleukin-1a gene expression is regulated by $\mathrm{Sp} 1$ and a transcriptional repressor. Cytokine 30, 141-153 (2005).

80. Alheim, K., McDowell, T. L., Symons, J. A., Duff, G. W. \& Bartfai, T. An AP-1 site is involved in the NGF induction of IL-1a in PC12 cells. Neurochem. Int. 29, 487-496 (1996).

81. Bailly, S., Fay, M., Israël, N. \& Gougerot-Pocidalo, M. A. The transcription factor AP-1 binds to the human interleukin 1 alpha promoter. Eur. Cytokine Netw. 7, 125-128 (1996).

82. Mori, N. \& Prager, D. Transactivation of the interleukin-1alpha promoter by human T-cell leukemia virus type I and type II Tax proteins. Blood 87, 3410-3417 (1996).

83. Chan, J. et al. A natural antisense transcript, AS-IL1a, controls inducible transcription of the pro-inflammatory cytokine IL-1a. J. Immunol. 195, 1359-1363 (2015). 
84. Laberge, R.-M. et al. MTOR regulates the pro-tumorigenic senescence-associated secretory phenotype by promoting IL1A translation. Nat. Cell Biol. 17, 1049-1061 (2015).

85. Dinarello, C. A. Interleukin-1 beta, interleukin-18, and the interleukin-1 beta converting enzyme. Ann. N. Y. Acad. Sci. 856, 1-11 (1998).

86. Liang, M. D., Zhang, Y., McDevit, D., Marecki, S. \& Nikolajczyk, B. S. The interleukin-1beta gene is transcribed from a poised promoter architecture in monocytes. J. Biol. Chem. 281, 9227-9237 (2006).

87. Husmann, M. et al. A novel SP-1 site in the human interleukin-1 beta promoter confers preferential transcriptional activity in keratinocytes. Eur. J. Immunol. 26, 3008-3014 (1996).

88. Yang, Z. et al. Dual regulatory role of human cytomegalovirus immediate-early protein in IL1B transcription is dependent upon Spi-1/PU.1. Biochem. Biophys. Res. Commun. 294, 854-863 (2002).

89. Yang, Z., Wara-Aswapati, N., Chen, C., Tsukada, J. \& Auron, P. E. NF-IL6 (C/ EBPbeta) vigorously activates il1b gene expression via a Spi-1 (PU.1) proteinprotein tether. J. Biol. Chem. 275, 21272-21277 (2000).

90. Kominato, Y., Galson, D., Waterman, W. R., Webb, A. C. \& Auron, P. E. Monocyte expression of the human prointerleukin 1 beta gene (IL1B) is dependent on promoter sequences which bind the hematopoietic transcription factor Spi-1/ PU.1. Mol. Cell Biol. 15, 58-68 (1995).

91. Tsukada, J., Saito, K., Waterman, W. R., Webb, A. C. \& Auron, P. E. Transcription factors NF-IL6 and CREB recognize a common essential site in the human prointerleukin 1 beta gene. Mol. Cell Biol. 14, 7285-7297 (1994).

92. Zhu, Q. \& Kanneganti, T.-D. Cutting edge: distinct regulatory mechanisms control proinflammatory cytokines IL-18 and IL-1ß. J. Immunol. 198, 4210-4215 (2017).

93. Puren, A. J., Fantuzzi, G. \& Dinarello, C. A. Gene expression, synthesis, and secretion of interleukin 18 and interleukin 1 beta are differentially regulated in human blood mononuclear cells and mouse spleen cells. Proc. Natl. Acad. Sci. USA 96, 2256-2261 (1999).

94. Marshall, J. D. et al. Regulation of human IL-18 mRNA expression. Clin. Immunol. 90, 15-21 (1999).

95. Gurram, V. C. et al. Genetic polymorphism of interleukin-18 gene promoter region in rheumatoid arthritis patients from southern India. J. Clin. Diagn. Res. 8 , SC01-SC04 (2014).

96. Thompson, S. R. \& Humphries, S. E. Interleukin-18 genetics and inflammatory disease susceptibility. Genes Immun. 8, 91-99 (2007).

97. Kim, Y. M. et al. Roles of IFN consensus sequence binding protein and PU.1 in regulating IL-18 gene expression. J. Immunol. 163, 2000-2007 (1999).

98. Kim, Y. M., Im, J. Y., Han, S. H., Kang, H. S. \& Choi, I. IFN-gamma up-regulates IL-18 gene expression via IFN consensus sequence-binding protein and activator protein-1 elements in macrophages. J. Immunol. 165, 3198-3205 (2000).

99. Kalina, U. et al. Genomic organization and regulation of the human interleukin18 gene. Scand. J. Immunol. 52, 525-530 (2000)

100. Takeda, N. et al. Bcl6 is a transcriptional repressor for the IL-18 gene. J. Immunol. 171, 426-431 (2003).

101. Koyama, N., Hoelzer, D. \& Ottmann, O. G. Regulation of human IL-18 gene expression: interaction of PU.1 with GC-box binding protein is involved in human IL-18 expression in myeloid cells. Eur. J. Immunol. 34, 817-826 (2004).

102. Roberts, T. L. et al. HIN-200 proteins regulate caspase activation in response to foreign cytoplasmic DNA. Science 323, 1057-1060 (2009).

103. Yang, J., Zhao, Y., Shi, J. \& Shao, F. Human NAIP and mouse NAIP1 recognize bacterial type III secretion needle protein for inflammasome activation. Proc. Natl. Acad. Sci. USA 110, 14408-14413 (2013).

104. Kofoed, E. M. \& Vance, R. E. Innate immune recognition of bacterial ligands by NAIPs determines inflammasome specificity. Nature 477, 592-595 (2011).

105. Suzuki, S. et al. Shigella type III secretion protein Mxil is recognized by Naip2 to induce Nlrc4 inflammasome activation independently of Pkcס. PLoS Pathog. 10, e1003926 (2014).

106. Zhao, Y. \& Shao, F. The NAIP-NLRC4 inflammasome in innate immune detection of bacterial flagellin and type III secretion apparatus. Immunol. Rev. 265, 85-102 (2015)

107. Zhao, Y. et al. The NLRC4 inflammasome receptors for bacterial flagellin and type III secretion apparatus. Nature 477, 596-600 (2011).

108. Man, S. M. et al. Inflammasome activation causes dual recruitment of NLRC4 and NLRP3 to the same macromolecular complex. Proc. Natl. Acad. Sci. USA 111, 7403-7408 (2014).

109. Matusiak, M. et al. Flagellin-induced NLRC4 phosphorylation primes the inflammasome for activation by NAIP5. Proc. Natl. Acad. Sci. USA 112, 1541-1546 (2015).

110. Qu, Y. et al. Phosphorylation of NLRC4 is critical for inflammasome activation. Nature 490, 539-542 (2012)
111. Tenthorey J. L. et al. New mutant mouse models clarify the role of NAIPs, phosphorylation, NLRP3, and tumors in NLRC4 inflammasome activation. bioRxiv https://doi.org/10.1101/765313 (2019).

112. Finger, J. N. et al. Autolytic proteolysis within the function to find domain (FIIND) is required for NLRP1 inflammasome activity. J. Biol. Chem. 287, 25030-25037 (2012).

113. $\mathrm{Xu}, \mathrm{H}$. et al. The $\mathrm{N}$-end rule ubiquitin ligase UBR2 mediates NLRP1B inflammasome activation by anthrax lethal toxin. EMBO J. 38, e101996 (2019).

114. Chui, A. J. et al. N-terminal degradation activates the NLRP1B inflammasome. Science 364, 82-85 (2019).

115. Sandstrom, A. et al. Functional degradation: a mechanism of NLRP1 inflammasome activation by diverse pathogen enzymes. Science $\mathbf{3 6 4}$, eaau1330 (2019).

116. Vasconcelos, N. M. et al. DPP8/DPP9 inhibition elicits canonical Nlrp1b inflammasome hallmarks in murine macrophages. Life Sci. Alliance 2, e201900313 (2019).

117. Okondo, M. C. et al. DPP8 and DPP9 inhibition induces pro-caspase-1dependent monocyte and macrophage pyroptosis. Nat. Chem. Biol. 13, 46-53 (2017).

118. Gai, K. et al. DPP8/9 inhibitors are universal activators of functional NLRP1 alleles. Cell Death Dis. 10, 587 (2019).

119. Park, Y. H., Wood, G., Kastner, D. L. \& Chae, J. J. Pyrin inflammasome activation and RhoA signaling in the autoinflammatory diseases FMF and HIDS. Nat. Immunol. 17, 914-921 (2016).

120. $\mathrm{Xu}, \mathrm{H}$. et al. Innate immune sensing of bacterial modifications of Rho GTPases by the Pyrin inflammasome. Nature 513, 237-241 (2014).

121. Mariathasan, S. et al. Cryopyrin activates the inflammasome in response to toxins and ATP. Nature 440, 228-232 (2006).

122. Hornung, V. et al. Silica crystals and aluminum salts activate the NALP3 inflammasome through phagosomal destabilization. Nat. Immunol. 9, 847-856 (2008).

123. Dostert, C. et al. Innate immune activation through Nalp3 inflammasome sensing of asbestos and silica. Science 320, 674-677 (2008).

124. Zhong, Z. et al. New mitochondrial DNA synthesis enables NLRP3 inflammasome activation. Nature 560, 198-203 (2018).

125. Zhong, Z. et al. TRPM2 links oxidative stress to NLRP3 inflammasome activation. Nat. Commun. 4, 1611 (2013).

126. Samir, P. et al. DDX3X acts as a live-or-die checkpoint in stressed cells by regulating NLRP3 inflammasome. Nature 573, 590-594 (2019).

127. Shi, H. et al. NLRP3 activation and mitosis are mutually exclusive events coordinated by NEK7, a new inflammasome component. Nat. Immunol. 17, 250-258 (2016).

128. He, Y., Zeng, M. Y., Yang, D., Motro, B. \& Núñez, G. NEK7 is an essential mediator of NLRP3 activation downstream of potassium efflux. Nature 530, 354-357 (2016).

129. Sharif, $H$. et al. Structural mechanism for NEK7-licensed activation of NLRP3 inflammasome. Nature 570, 338-343 (2019).

130. Pétrilli, V. et al. Activation of the NALP3 inflammasome is triggered by low intracellular potassium concentration. Cell Death Differ. 14, 1583-1589 (2007).

131. Muñoz-Planillo, R. et al. $\mathrm{K}+$ efflux is the common trigger of NLRP3 inflammasome activation by bacterial toxins and particulate matter. Immunity 38, 1142-1153 (2013).

132. Groß, C. J. et al. K+ efflux-independent NLRP3 inflammasome activation by small molecules targeting mitochondria. Immunity 45, 761-773 (2016).

133. Misawa, T. et al. Microtubule-driven spatial arrangement of mitochondria promotes activation of the NLRP3 inflammasome. Nat. Immunol. 14, 454-460 (2013).

134. Liu, Q., Zhang, D., Hu, D., Zhou, X. \& Zhou, Y. The role of mitochondria in NLRP3 inflammasome activation. Mol. Immunol. 103, 115-124 (2018).

135. Chen, J. \& Chen, Z. J. Ptdlns4P on dispersed trans-Golgi network mediates NLRP3 inflammasome activation. Nature 564, 71-76 (2018).

136. Kayagaki, N. et al. Non-canonical inflammasome activation targets caspase- 11 . Nature 479, 117-121 (2011).

137. He, X. et al. Inflammation and fibrosis during Chlamydia pneumoniae infection is regulated by $\mathrm{IL}-1$ and the NLRP3/ASC inflammasome. J. Immunol. 184, 5743-5754 (2010).

138. Harder, J. et al. Activation of the Nlrp3 inflammasome by Streptococcus pyogenes requires Streptolysin O and NF-KB activation but proceeds independently of TLR signaling and P2X7 receptor. J. Immunol. 183, 5823-5829 (2009).

139. Mishra, B. B. et al. Mycobacterium tuberculosis protein ESAT-6 is a potent activator of the NLRP3/ASC inflammasome. Cell Microbiol. 12, 1046-1063 (2010).

140. Karki, R. et al. Concerted activation of the AIM2 and NLRP3 inflammasomes orchestrates host protection against aspergillus infection. Cell Host Microbe 17, 357-368 (2015). 
141. Hagar, J. A., Powell, D. A., Aachoui, Y., Ernst, R. K. \& Miao, E. A. Cytoplasmic LPS activates caspase-11: implications in TLR4-independent endotoxic shock. Science 341, 1250-1253 (2013).

142. Shi, J. et al. Inflammatory caspases are innate immune receptors for intracellular LPS. Nature 514, 187-192 (2014).

143. Rühl, S. \& Broz, P. Caspase-11 activates a canonical NLRP3 inflammasome by promoting $\mathrm{K}^{+}$efflux. Eur. J. Immunol. 45, 2927-2936 (2015).

144. Kuriakose, T. \& Kanneganti, T.-D. ZBP1: innate sensor regulating cell death and inflammation. Trends Immunol. 39, 123-134 (2018).

145. Kesavardhana, S. et al. ZBP1/DAl ubiquitination and sensing of influenza vRNPs activate programmed cell death. J. Exp. Med. 214, 2217-2229 (2017).

146. Thapa, R. J. et al. DAl senses influenza A virus genomic RNA and activates RIPK3dependent cell death. Cell Host Microbe 20, 674-681 (2016).

147. Malireddi, R. K. S. et al. TAK1 restricts spontaneous NLRP3 activation and cell death to control myeloid proliferation. J. Exp. Med. 215, 1023-1034 (2018).

148. Sarhan, J. et al. Caspase-8 induces cleavage of gasdermin D to elicit pyroptosis during Yersinia infection. Proc. Natl. Acad. Sci. USA 115, E10888-E10897 (2018).

149. Orning, P. et al. Pathogen blockade of TAK1 triggers caspase-8-dependent cleavage of gasdermin D and cell death. Science 362, 1064-1069 (2018).

150. Chen, K. W. et al. Extrinsic and intrinsic apoptosis activate pannexin-1 to drive NLRP3 inflammasome assembly. EMBO J. 38, e101638 (2019).

151. Malireddi, R. K. S. et al. Innate immune priming in the absence of TAK1 drives RIPK1 kinase activity-independent pyroptosis, apoptosis, necroptosis, and inflammatory disease. J. Exp. Med. 217, e20191644 (2020)

152. Malireddi, R. K. S., Kesavardhana, S. \& Kanneganti, T.-D. ZBP1 and TAK1: master regulators of NLRP3 inflammasome/pyroptosis, apoptosis, and necroptosis (PAN-optosis). Front. Cell Infect. Microbiol. 9, 406 (2019).

153. Sborgi, L. et al. Structure and assembly of the mouse ASC inflammasome by combined NMR spectroscopy and cryo-electron microscopy. Proc. Natl. Acad. Sci. USA 112, 13237-13242 (2015).

154. Lu, A. et al. Unified polymerization mechanism for the assembly of ASC dependent inflammasomes. Cell 156, 1193-1206 (2014).

155. Dick, M. S., Sborgi, L., Rühl, S., Hiller, S. \& Broz, P. ASC filament formation serves as a signal amplification mechanism for inflammasomes. Nat. Commun. 7, 11929 (2016).

156. Ferrao, R. \& Wu, H. Helical assembly in the death domain (DD) superfamily. Curr. Opin. Struct. Biol. 22, 241-247 (2012).

157. Park, H. H. et al. The death domain superfamily in intracellular signaling of apoptosis and inflammation. Annu. Rev. Immunol. 25, 561-586 (2007).

158. Wu H. \& Lo, Y.-C. Structures, domains and functions in cell death (DD, DED, CARD, PYD). In: Encyclopedia of LifeSciences (ELS). John Wiley \& Sons, Ltd: Chichester. https://doi.org/10.1002/9780470015902.a0021579 (2009).

159. Kagan, J. C., Magupalli, V. G. \& Wu, H. SMOCs: supramolecular organizing centres that control innate immunity. Nat. Rev. Immunol. 14, 821-826 (2014).

160. Qiao, Q. \& Wu, H. Supramolecular organizing centers (SMOCs) as signaling machines in innate immune activation. Sci. China Life Sci. 58, 1067-1072 (2015).

161. Lu, A. et al. Molecular basis of caspase- 1 polymerization and its inhibition by a new capping mechanism. Nat. Struct. Mol. Biol. 23, 416-425 (2016).

162. Lu, A. et al. Plasticity in PYD assembly revealed by cryo-EM structure of the PYD filament of AIM2. Cell Discov. 1, 15013 (2015).

163. Zhang, L. et al. Cryo-EM structure of the activated NAIP2-NLRC4 inflammasome reveals nucleated polymerization. Science 350, 404-409 (2015).

164. Hu, Z. et al. Structural and biochemical basis for induced self-propagation of NLRC4. Science 350, 399-404 (2015).

165. Jin, T. et al. Structures of the HIN domain: DNA complexes reveal ligand binding and activation mechanisms of the AIM2 inflammasome and IFI16 receptor. Immunity 36, 561-571 (2012).

166. Yin, Q. et al. Molecular mechanism for p202-mediated specific inhibition of AIM2 inflammasome activation. Cell Rep. 4, 327-339 (2013).

167. Tenthorey, J. L. et al. The structural basis of flagellin detection by NAIP5: a strategy to limit pathogen immune evasion. Science 358, 888-893 (2017).

168. Hu, Z. et al. Crystal structure of NLRC4 reveals its autoinhibition mechanism. Science 341, 172-175 (2013).
169. Ruan, J., Xia, S., Liu, X., Lieberman, J. \& Wu, H. Cryo-EM structure of the gasdermin A3 membrane pore. Nature 557, 62-67 (2018).

170. Mulvihill, E. et al. Mechanism of membrane pore formation by human gasdermin-D. EMBO J. 37, e98321 (2018).

171. Segovia, M. et al. Targeting TMEM176B enhances antitumor immunity and augments the efficacy of immune checkpoint blockers by unleashing inflammasome activation. Cancer Cell 35, 767-781.e6 (2019).

172. Ridker, P. M., Thuren, T., Zalewski, A. \& Libby, P. Interleukin-1 $\beta$ inhibition and the prevention of recurrent cardiovascular events: Rationale and Design of the Canakinumab Anti-inflammatory Thrombosis Outcomes Study (CANTOS). Am. Heart J. 162, 597-605 (2011).

173. Ridker, P. M. et al. Effect of interleukin-1 $\beta$ inhibition with canakinumab on incident lung cancer in patients with atherosclerosis: exploratory results from a randomised, double-blind, placebo-controlled trial. The Lancet 390, 1833-1842 (2017).

174. Qu, Y. et al. NLRP3 recruitment by NLRC4 during Salmonella infection. J. Exp. Med. 213, 877-885 (2016).

175. Stutz, A. et al. NLRP3 inflammasome assembly is regulated by phosphorylation of the pyrin domain. J. Exp. Med. 214, 1725-1736 (2017).

176. Song, N. et al. NLRP3 phosphorylation is an essential priming event for inflammasome activation. Mol. Cell 68, 185-197.e6 (2017).

177. Zhang, Z. et al. Protein kinase D at the Golgi controls NLRP3 inflammasome activation. J. Exp. Med. 214, 2671-2693 (2017).

178. Mortimer, L., Moreau, F., MacDonald, J. A. \& Chadee, K. NLRP3 inflammasome inhibition is disrupted in a group of auto-inflammatory disease CAPS mutations. Nat. Immunol. 17, 1176-1186 (2016).

179. Guo, C. et al. Bile Acids Control Inflammation and Metabolic Disorder through Inhibition of NLRP3 Inflammasome. Immunity 45, 944 (2016).

180. Spalinger, M. R. et al. NLRP3 tyrosine phosphorylation is controlled by protein tyrosine phosphatase PTPN22. J. Clin. Investig. 126, 4388 (2016)

181. Py, B. F., Kim, M.-S., Vakifahmetoglu-Norberg, H. \& Yuan, J. Deubiquitination of NLRP3 by BRCC3 critically regulates inflammasome activity. Mol. Cell 49, 331-338 (2013).

182. Han, S. et al. Lipopolysaccharide primes the NALP3 inflammasome by inhibiting its ubiquitination and degradation mediated by the SCFFBXL2 E3 ligase. J. Biol. Chem. 290, 18124-18133 (2015).

183. Song, $\mathrm{H}$. et al. The E3 ubiquitin ligase TRIM31 attenuates NLRP3 inflammasome activation by promoting proteasomal degradation of NLRP3. Nat. Commun. 7, 13727 (2016).

184. Yan, Y. et al. Dopamine controls systemic inflammation through inhibition of NLRP3 inflammasome. Cell 160, 62-73 (2015).

185. Kawashima, A. et al. ARIH2 ubiquitinates NLRP3 and negatively regulates NLRP3 inflammasome activation in macrophages. J. Immunol. 199, 3614-3622 (2017).

186. Cocco, M. et al. Design, synthesis, and evaluation of acrylamide derivatives as direct NLRP3 inflammasome inhibitors. ChemMedChem 11, 1790-1803 (2016).

187. Mishra, B. B. et al. Nitric oxide controls the immunopathology of tuberculosis by inhibiting NLRP3 inflammasome-dependent processing of IL-1 $\beta$. Nat. Immunol. 14, 52-60 (2013).

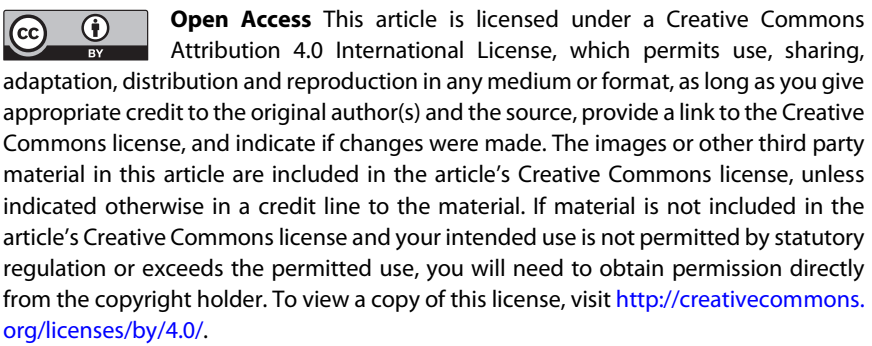

(c) The Author(s) 2020 